\title{
Supplementary materiel to "North Atlantic Oscillation controls on oxygen and hydrogen isotope gradients in winter precipitation across Europe; implications for palaeoclimate studies"
}

\author{
Michael Deininger ${ }^{1}$, Martin Werner ${ }^{2}$, Frank McDermott ${ }^{1}$ \\ ${ }^{1}$ UCD School of Earth Sciences, University College Dublin, Belfield, Dublin 4, Ireland \\ ${ }^{2}$ Alfred Wegener Institute, Helmholtz Centre for Polar and Marine Research, Bussestraße 24, 27570 \\ Bremerhaven, Germany \\ Correspondence to: Michael Deininger (michael.deininger@ucd.ie)
}


Multi-box exercise. The effect of the precipitation history on a longitudinal gradient, is illustrated in Figure $\mathrm{S} 1$ for $\delta^{18} \mathrm{O}_{\mathrm{P}}$ using a multi-box Rayleigh distillation model. The multi-box model is composed of 15 boxes. The initial $\delta^{18} \mathrm{O}$ value of the atmospheric moisture in the $1^{\text {st }}$ box is assumed to be $-20 \%$. The $\delta^{18} \mathrm{O}_{\mathrm{P}}$ value and the $\delta^{18} \mathrm{O}$ value of the atmospheric moisture of each box is calculated using the remaining amount of atmospheric moisture and its $\delta^{18} \mathrm{O}$ value (transferred from the previous box), the respective amount of rainfall and the temperature at condensation using the standard Rayleigh equations for the mother and daughter products (Mook, 2006) and the isotope fractionation factor of Majoube (1971). The temperature and the amount of precipitation in every box is kept constant at $10^{\circ} \mathrm{C}$ and $10 \mathrm{~mm}$, respectively. To vary the amount of precipitable water $\mathrm{Q}_{0}$ equates the sum of the total precipitation in all boxes and is multiplied by a factor that varies between 2.5 and 1.3. The higher the factor is the more moisture is left in the last box. The multi-box approach demonstrates that $\delta^{18} \mathrm{O}_{\mathrm{P}}$ values are more sensitive to the precipitation history $\mathrm{f}$ when the initial amount of precipitable water $\mathrm{Q}_{0}$ is smaller. Similar results are obtained if instead of $\mathrm{Q}_{0}$ the amount of precipitation is varied resulting in steeper longitudinal gradients if the amount of precipitation increases (not shown).

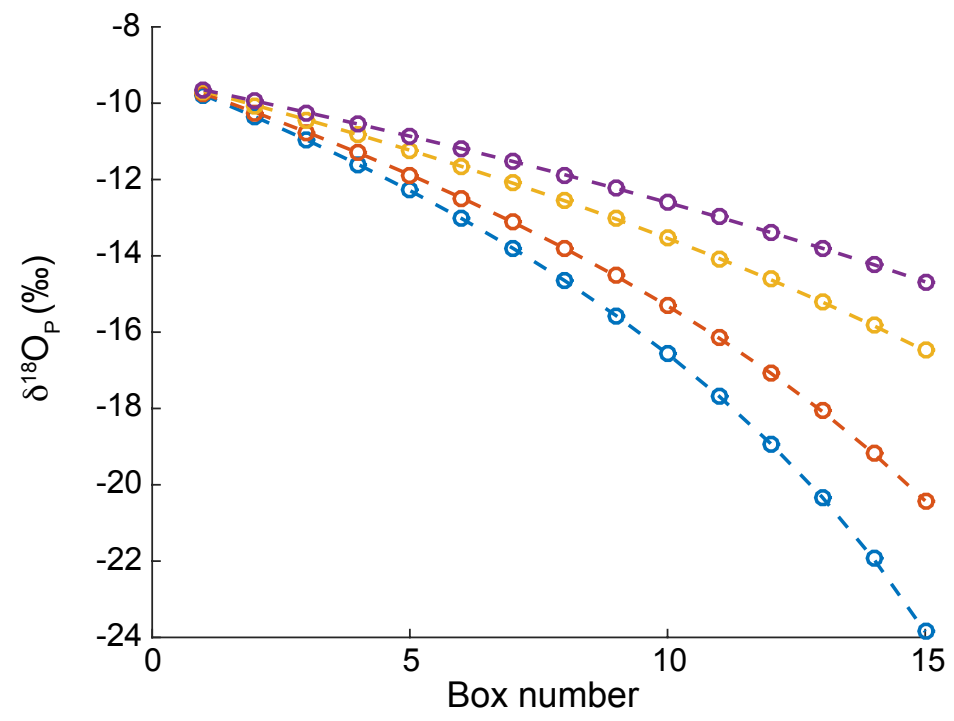

Figure S1: Multi-box exercise assuming a Rayleigh-type model for the condensation and associated isotope fractionation for precipitation $\delta^{18} \mathrm{OP}$. The propagation of the $\delta^{18} \mathrm{O}_{\mathrm{P}}$ values through the boxes for different initial amounts of atmospheric moisture $\mathrm{Q}_{0} . \mathrm{Q}_{0}$ is determined in times of total precipitation of all boxes with a scaling factor of 1.3 (blue), 1.5 (orange), 2 (yellow) and 2.5 (purple) showing that the $\delta^{18} \mathrm{O}_{\mathrm{P}}$ values along the transect (from box to 1 to 15 ) becomes more depleted the smaller $\mathrm{Q}_{0}$ is. Therefore, a longitudinal transect of $\delta^{18} \mathrm{O}_{\mathrm{P}}\left(\right.$ and $\delta \mathrm{D}_{\mathrm{P}}$ ) values is more sensitive to the precipitation history, in terms of the isotope depletion in rare ${ }^{18} \mathrm{O}$ and ${ }^{2} \mathrm{H}$ isotopes along a longitudinal gradient when $\mathrm{Q}_{0}$ is smaller resulting in steeper longitudinal $\delta^{18} \mathrm{O}_{\mathrm{P}}$ (and $\delta \mathrm{D}_{\mathrm{P}}$ ) gradients, i.e., increasing gradient slopes. 


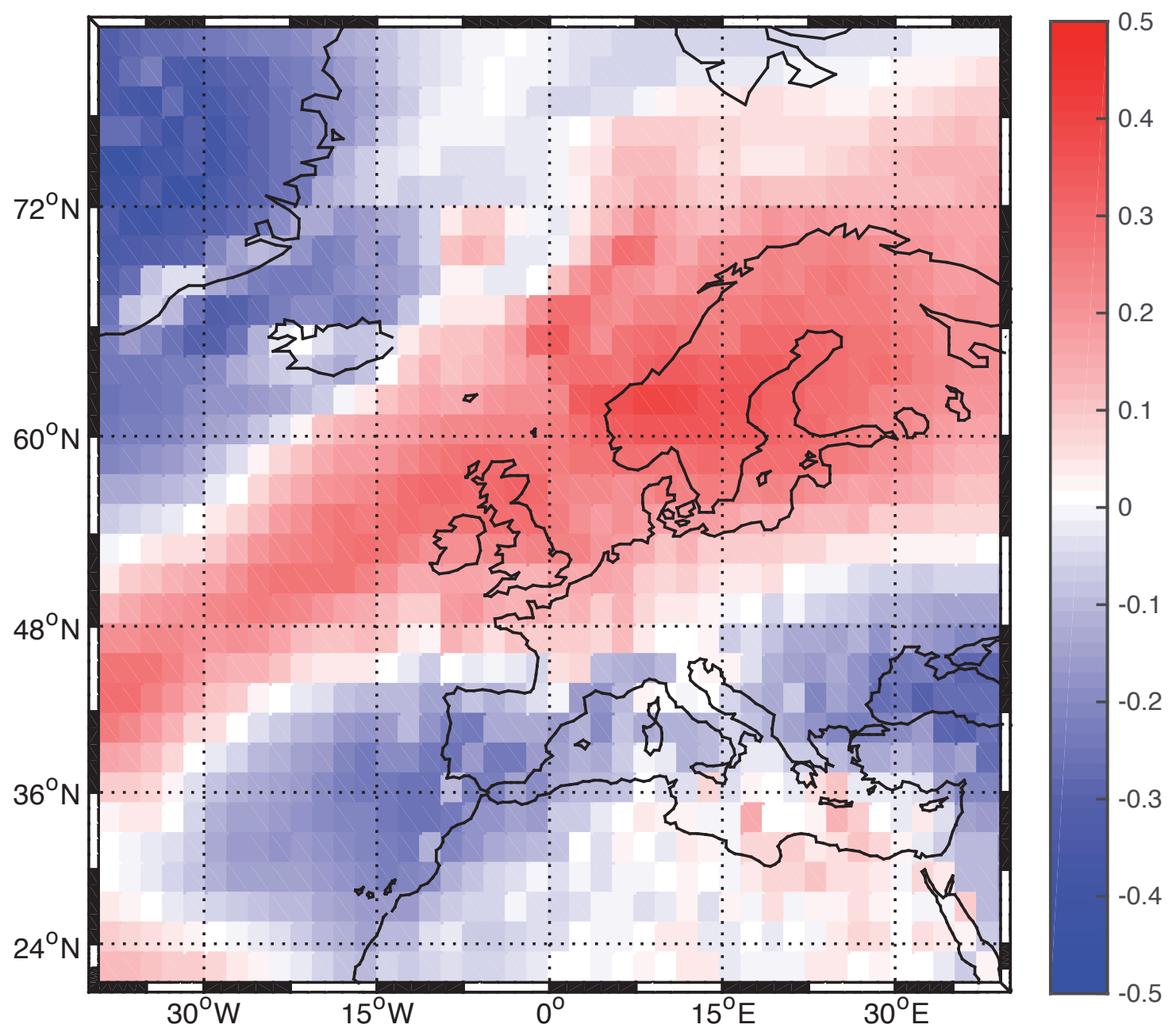

Figure S2: Correlation map between the monthly index of the North Atlantic Oscillation and the amount of total precipitable water for the month from December to March derived from ECHAM5-wiso simulation. The map shows the sector from $20^{\circ} \mathrm{N}$ to $80^{\circ} \mathrm{N}$ and from $40^{\circ} \mathrm{W}$ to $40^{\circ} \mathrm{E}$ for the years from 1960 to $2010 \mathrm{AD}$ (see text for details on the ECAHM5-wiso simulations). The colour bar on the right side indicates the colour code for the correlation coefficients. 
Supplementary discussion on the mechanisms that control the $\delta^{18} \mathrm{O}_{\mathrm{pw}}$ and $\delta \mathrm{D}_{\mathrm{pw}}$ of the Mediterranean stations. There are clear relationships (for the 3-month and 6-month winter period) of temperature $(r>0.5)$ and precipitation $(\mathrm{r}<-0.5)$ to the wNAOi as well as between these two parameters and $\delta^{18} \mathrm{O}_{\mathrm{pw}}$ and $\delta \mathrm{D}_{\mathrm{pw}}$ $(\mathrm{r}>0.5$ for temperature and $\mathrm{r}<-0.4$ for precipitation) from the station Avignon suggesting that temperature and precipitation are important parameter that control $\delta^{18} \mathrm{O}_{\mathrm{pw}}$ and $\delta \mathrm{D}_{\mathrm{pw}}$. For Zagreb in contrast the controlling mechanism for the observed strong NAO-relationship remain unclear. For the 3-month dataset there is a NAO-relationship to temperature $(r>0.45)$ and precipitation $(r<-0.5)$ but this is clearly absent for the 6-month dataset. Similar relationships between temperature and precipitation and the $\delta^{18} \mathrm{O}_{\mathrm{pw}}$ and $\delta \mathrm{D}_{\mathrm{pw}}$ datasets as for Avignon are observed for the 3-month dataset $(r>0.3$ for temperature and $r<-0.4$ for precipitation). For Locarno a NAO-relationship is found for the temperature $(r>0.45)$ as well as the precipitation $(\mathrm{r}<-0.33)$ for both the 3-month and 6-month winter period. For $\delta^{18} \mathrm{O}_{\mathrm{pw}}$ only a relationship to the temperature is observed $(r>0.5)$ while there is a relationship between $\delta D_{p w}$ and temperature $(r>0.4)$ and precipitation $(r>0.45)$. For the temperature and precipitation datasets from Genoa (Setri) only the precipitation has a relationship to the wNAOi $(\mathrm{r}<-0.4)$. However, there are no relationships observed between temperature and precipitation and the $\delta^{18} \mathrm{O}_{\mathrm{pw}}$ and $\delta \mathrm{D}_{\mathrm{pw}}$ datasets; only the 3-month $\delta \mathrm{D}_{\mathrm{pw}}$ dataset has a relationship to the temperature $(\mathrm{r}=0.38)$. 
Supplementary data. The left panels show the box plots for the 6 months (October to March) and the right panels for the 3 months (December to February) winter season. The upper panels illustrate the $\delta^{18} \mathrm{O}_{\mathrm{pw}}$ values and the lower panels the $\delta \mathrm{D}_{\mathrm{pw}}$ values. Every box plot illustrates the statistical variables (median, min, max, 25 and $75 \%$ quantile) for every wNAOi class from lowest to highest (left to right). Note that for some stations no data was available for NAO classes; in these cases the statistical results are only illustrated for the classes where data was collected (e.g. Station 15, Bern). For the individual wNAOi classes, the red line illustrates the median of the compilation of data; the edges of the blue rectangles marks the $25 \%$ and $75 \%$ quantile; the black bars illustrates the minimum and maximum value and the red plus sign pictures outliers (maximum whisker length is 1.5). (The figures were plotted using the MATLAB ${ }^{\circledR}$ function "boxplot".)

\section{Station 1: Valentia (OBSERVATORY)}

a)

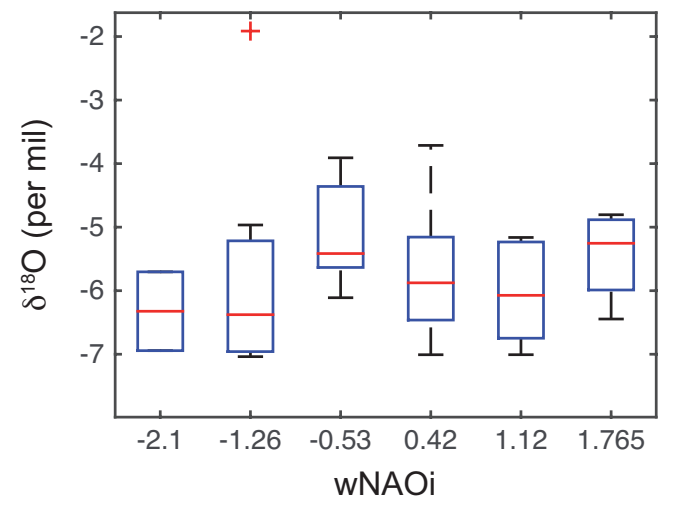

c)

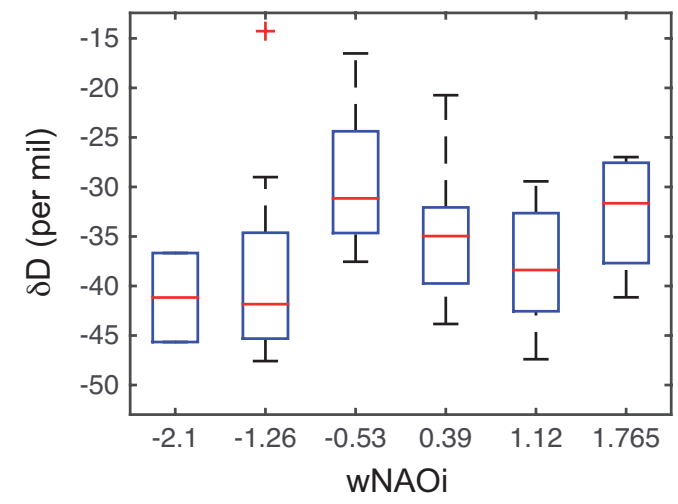

b)

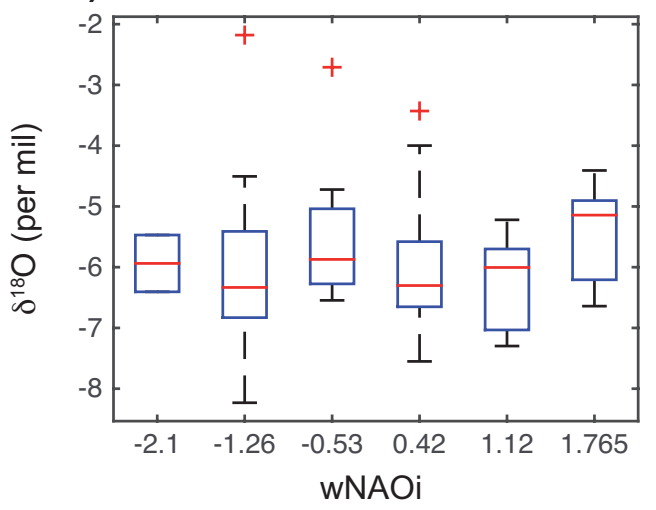

d)

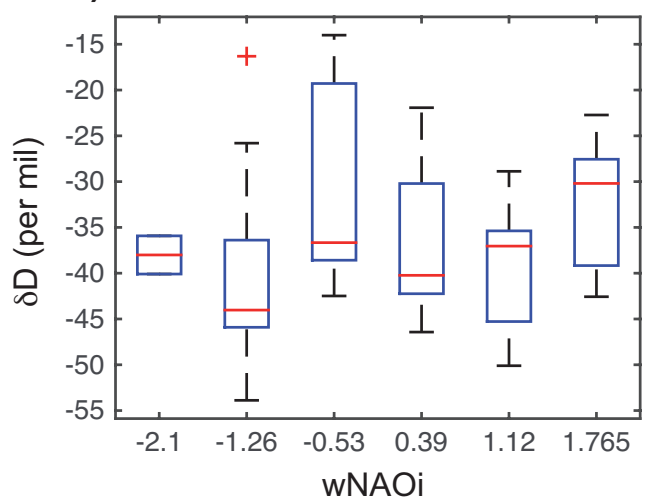

Figure caption exemplary given for the Valentia (OBSERVATORY): box plot (as described above) for the 6 months winter season (October to March) a) $\delta^{18} \mathrm{O}_{\mathrm{pw}}$ and c) $\delta \mathrm{D}_{\mathrm{pw}}$ values and for the 3 months winter season (December to February) b) $\delta^{18} \mathrm{O}_{\mathrm{pw}}$ and d) $\delta \mathrm{D}_{\mathrm{pw}}$ values for the various wNAOi classes sorted from the lowest (left) to the highest (right) wNAOi class. The red line illustrates the median; the edges of the blue rectangles marks the $25 \%$ and $75 \%$ quantile; the black bars illustrates the minimum and maximum value and the red plus sign pictures outliers. 
Station 2: Wallingford

a)

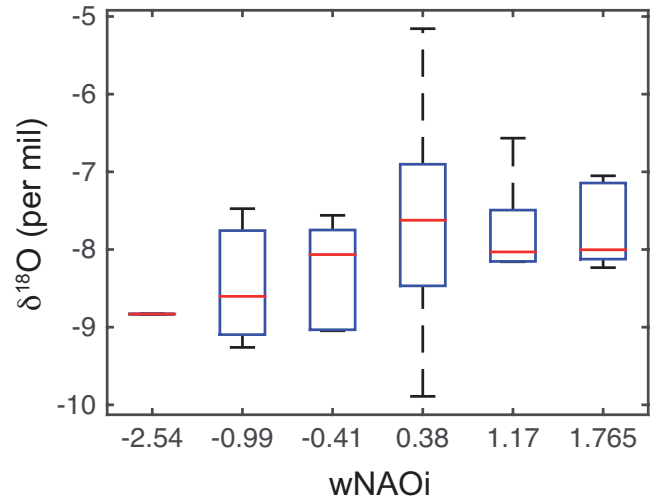

c)

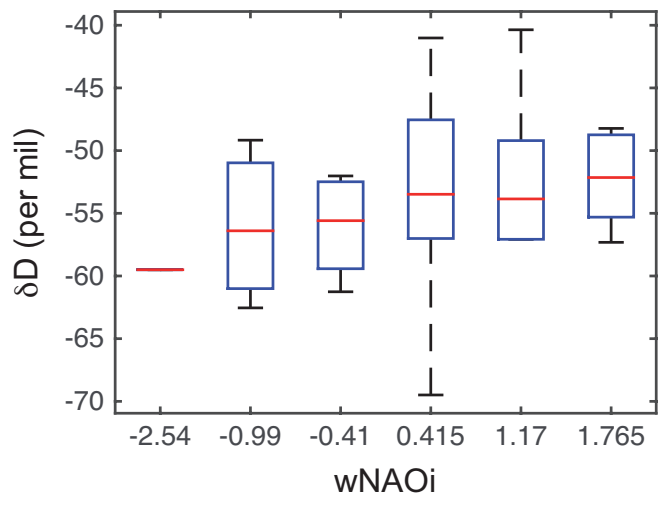

b)

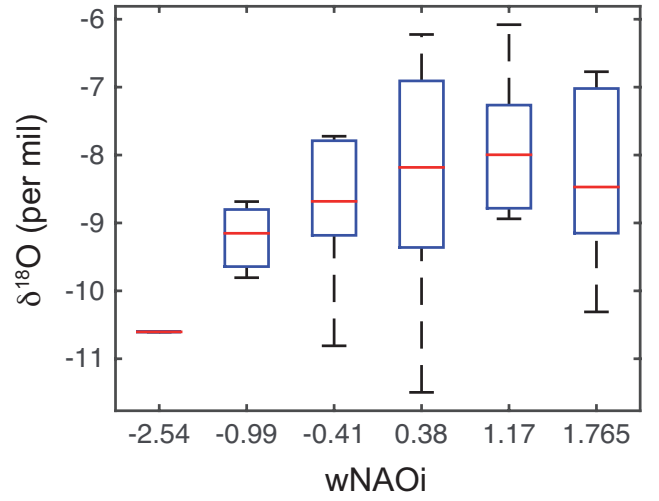

d)

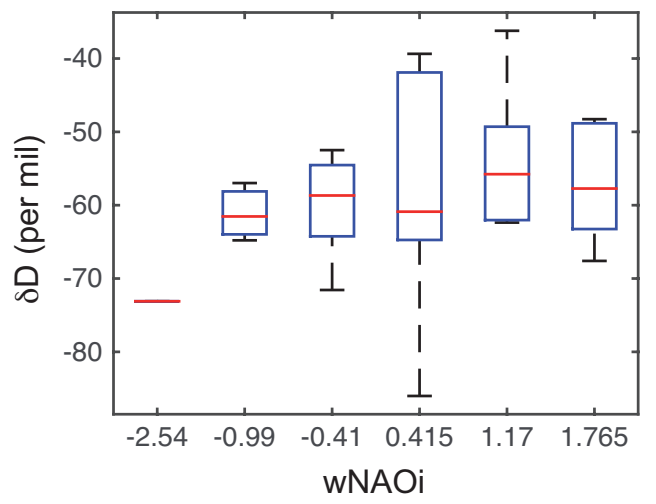

\section{Station 3: Groningen}


a)

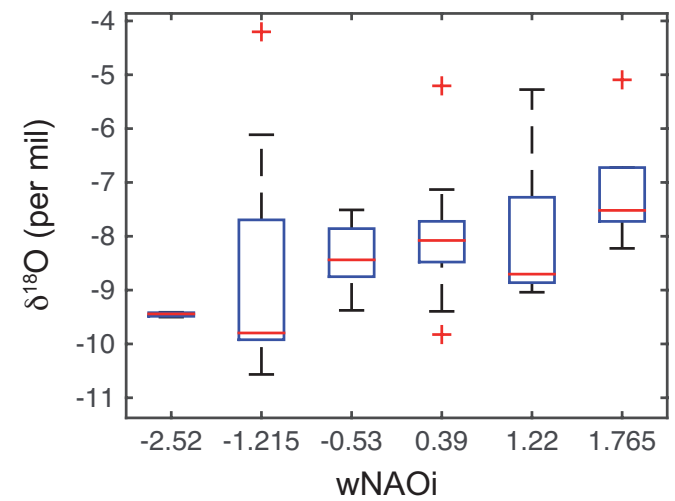

\section{c)}

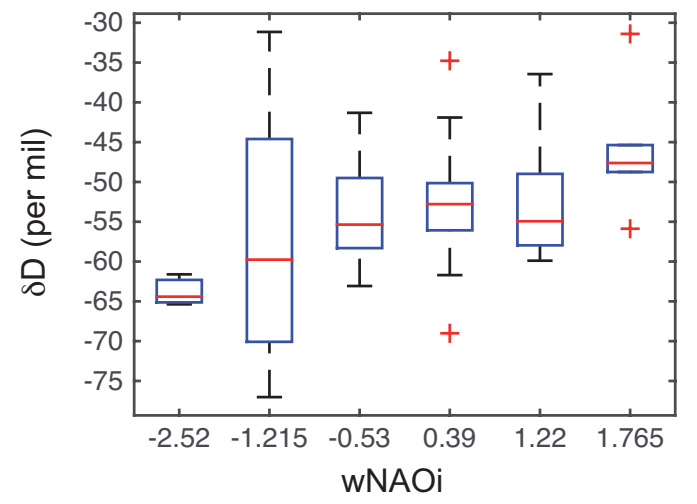

b)

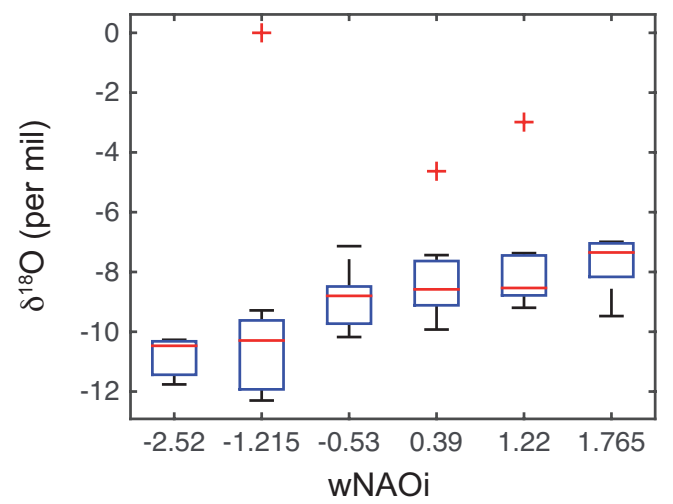

d)

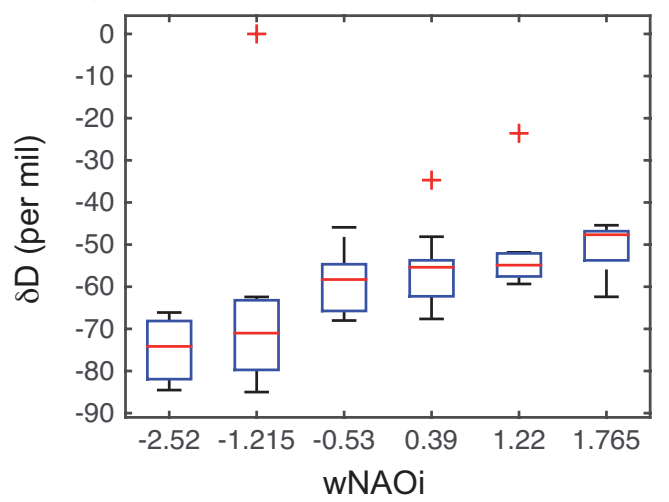

\section{Station 4: Koblenz}


a)

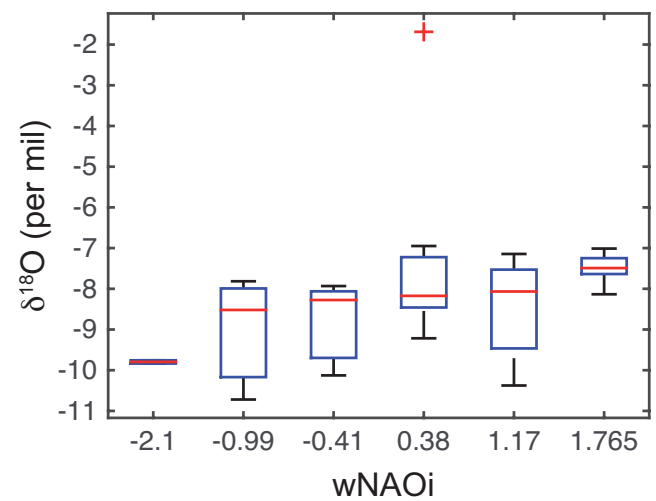

c)

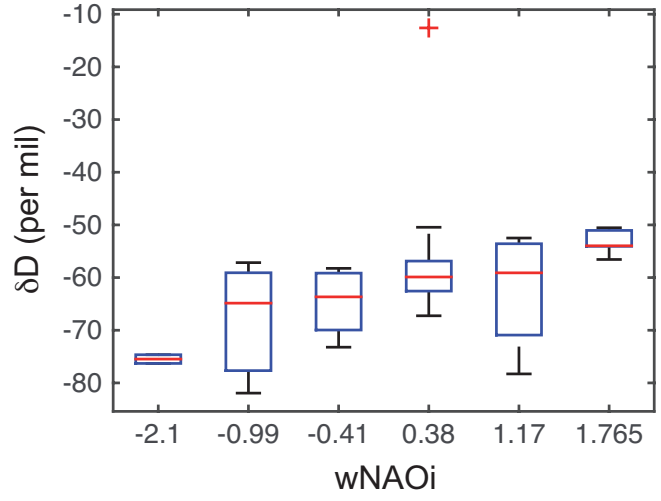

b)

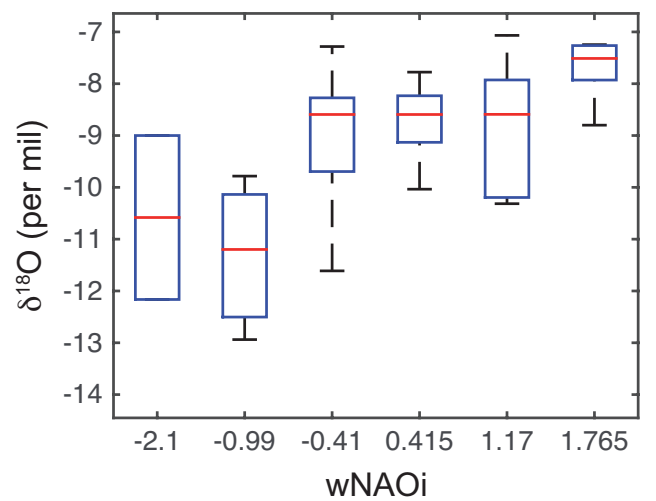

d)

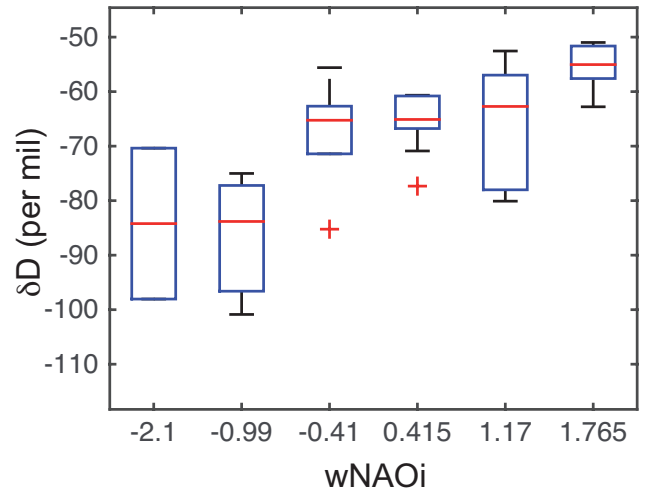

Station 5: Karlsruhe 
a)

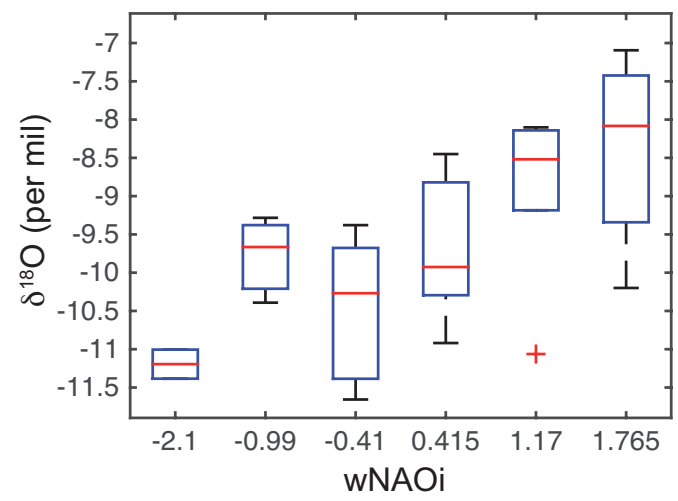

c)

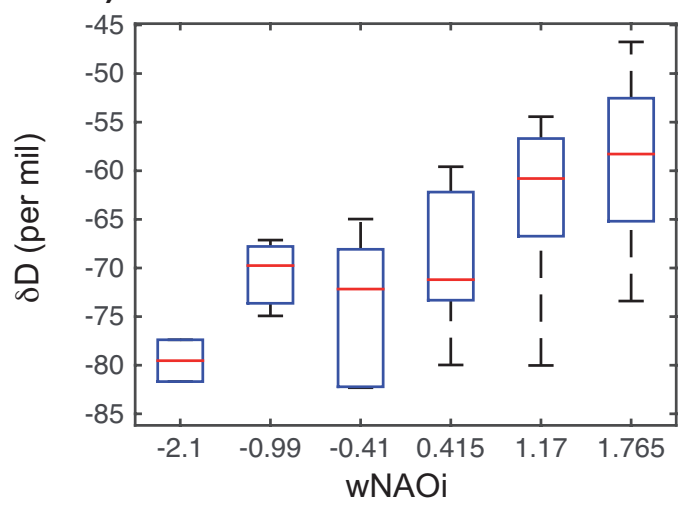

b)

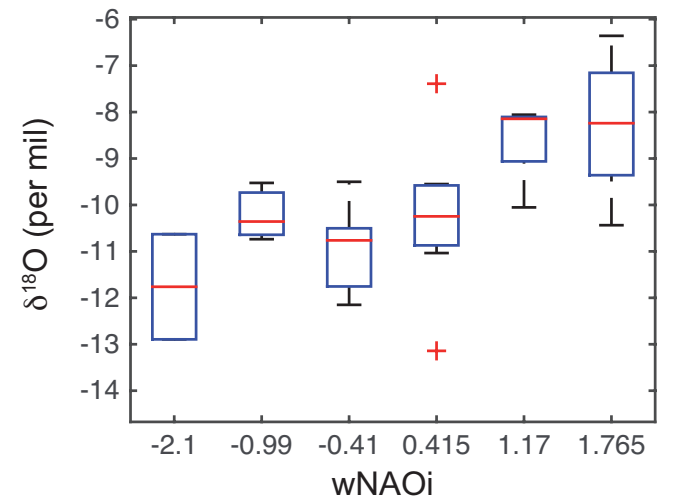

d)

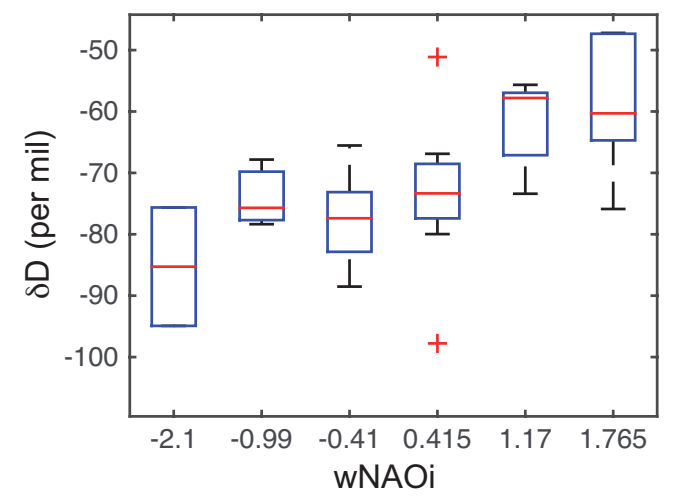

Station 6: Cuxhaven 
a)

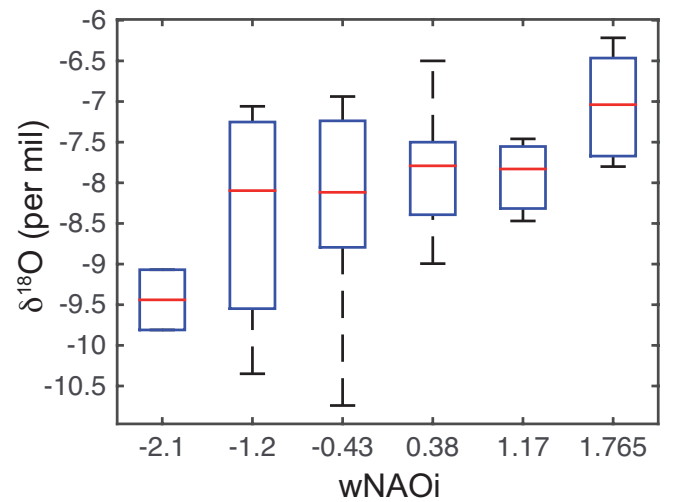

c)

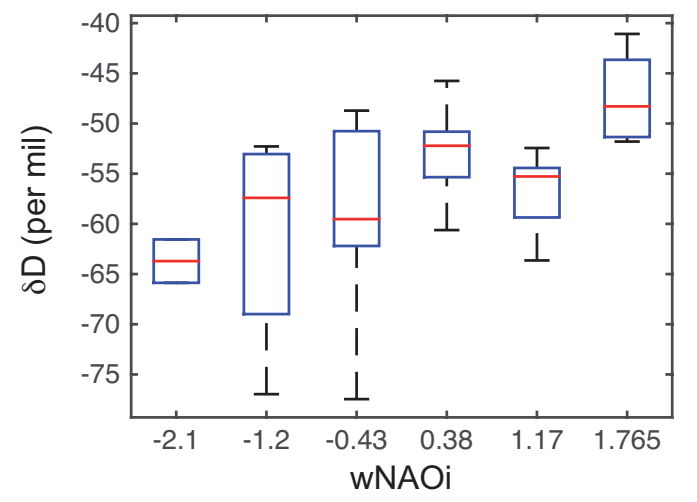

b)

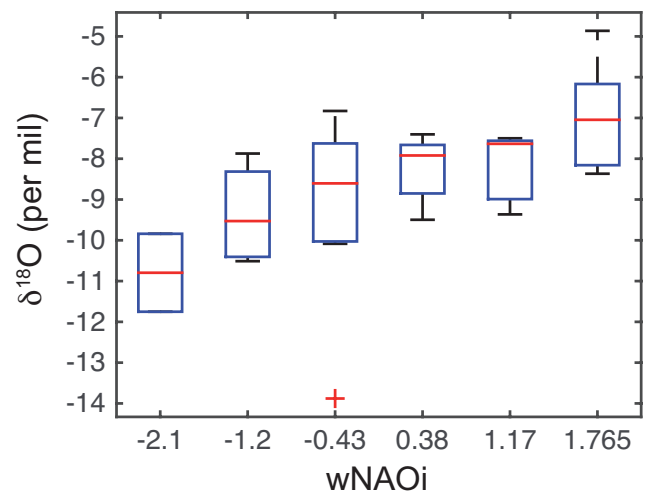

d)

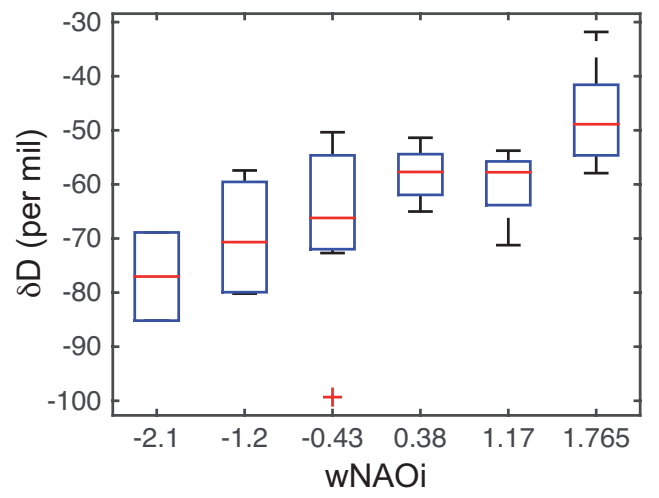

Station 7: Bad Salzuflen 
a)

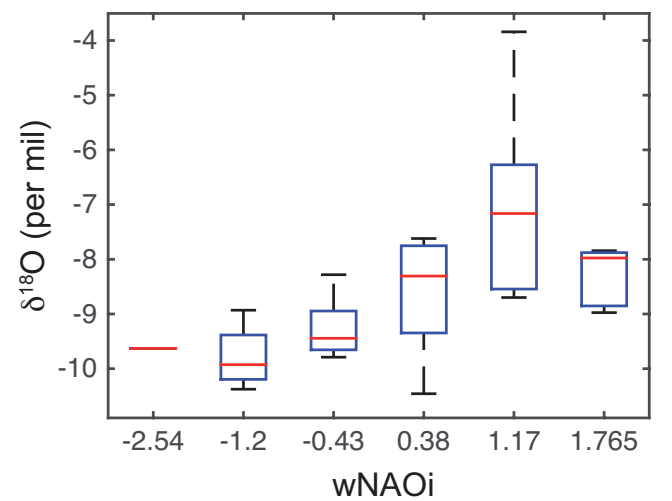

c)

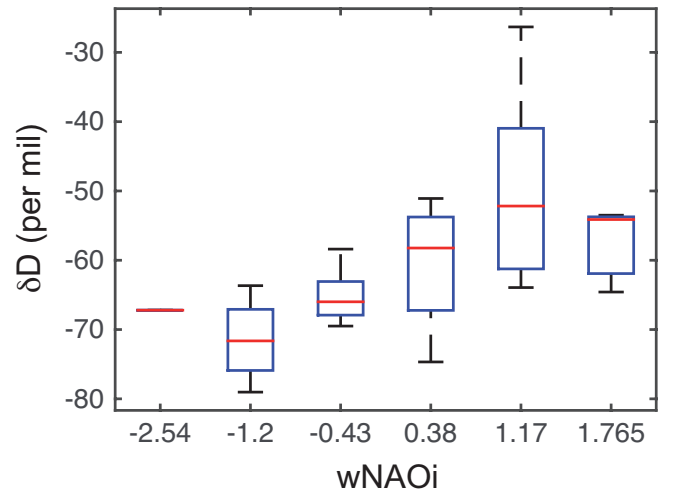

b)

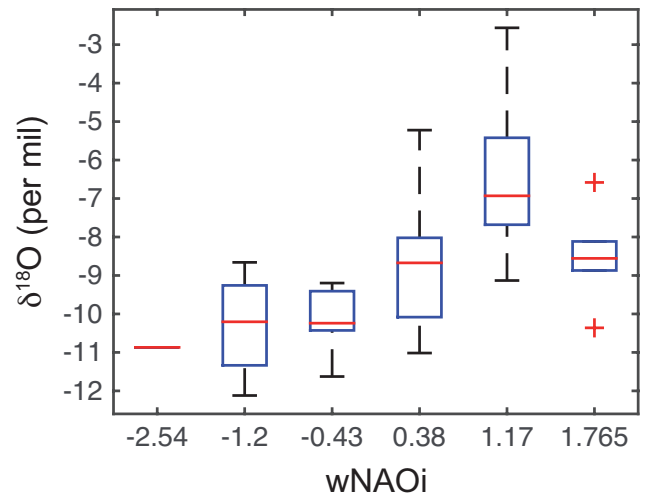

d)

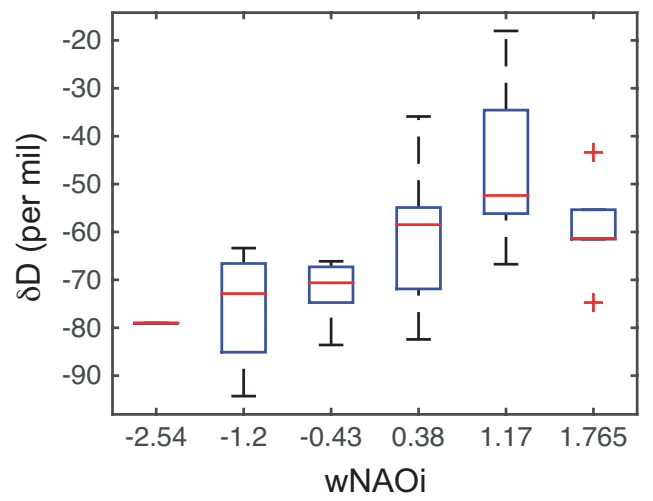

Station 8: Stuttgart (CANNSTATT) 
a)

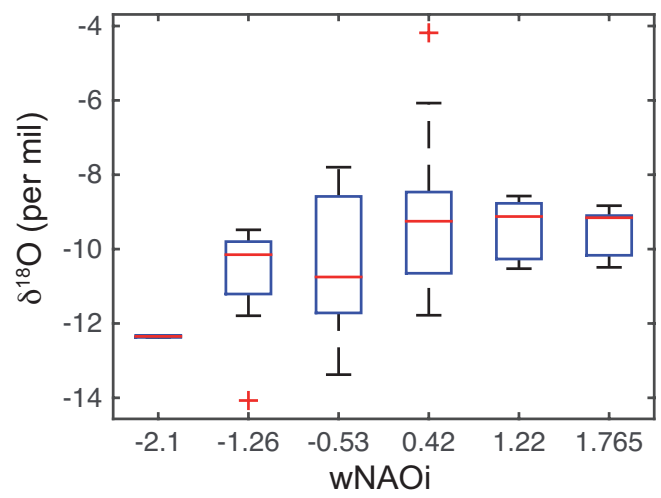

c)

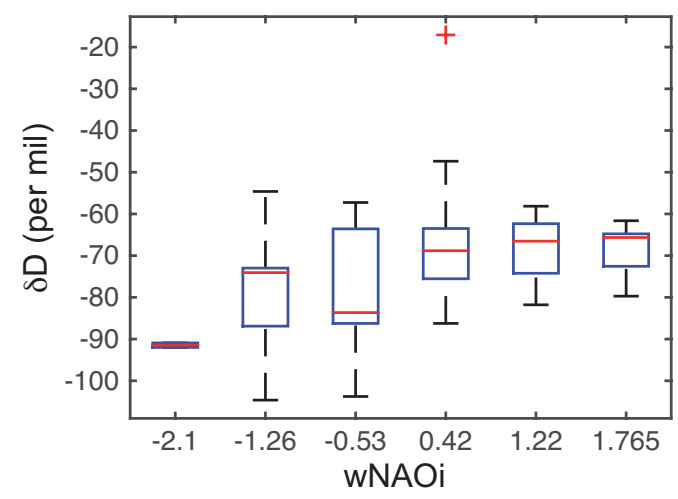

b)

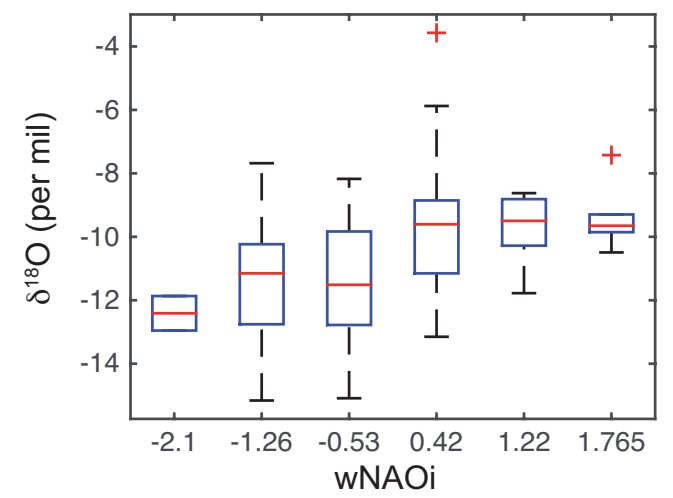

d)

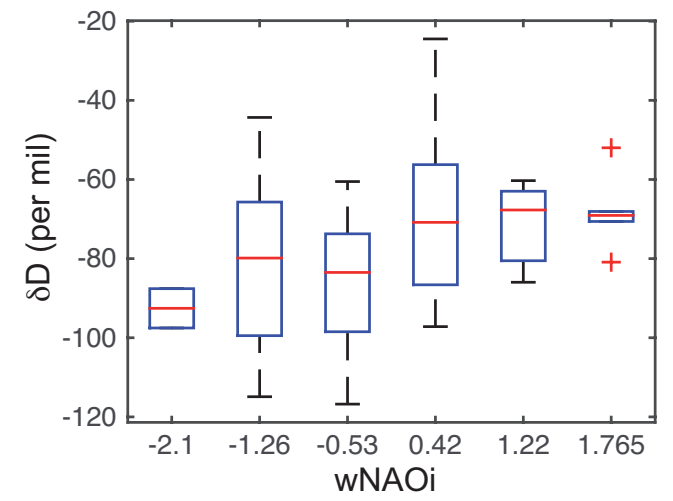

\section{Station 9: Wuerzburg}


a)

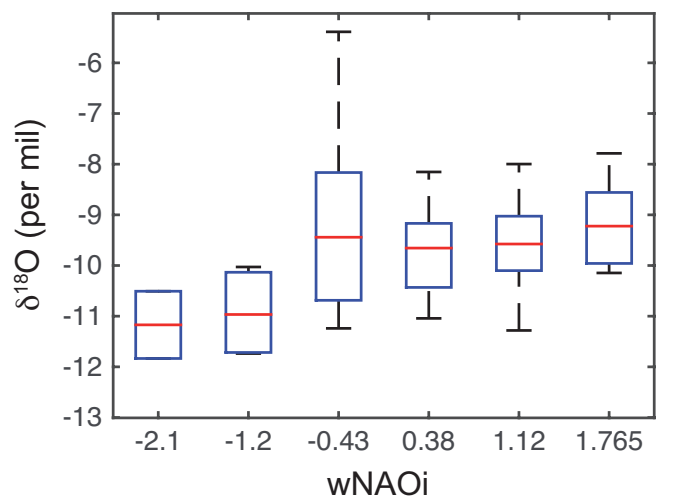

c)

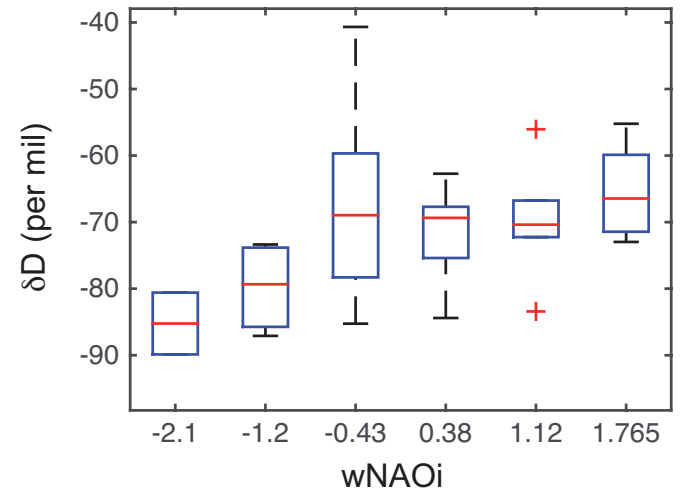

b)

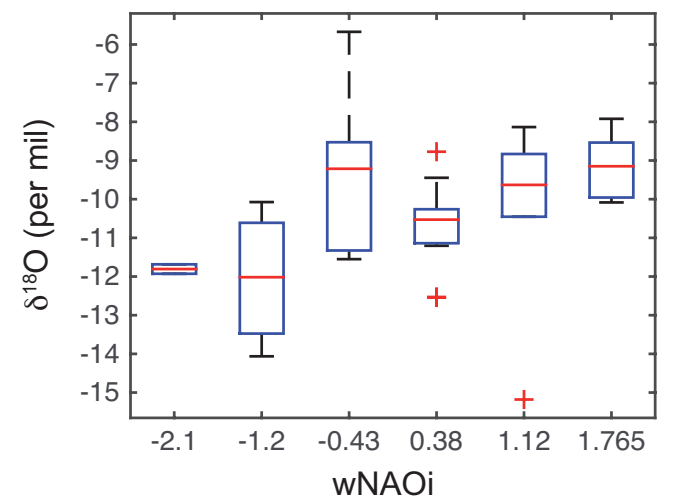

d)

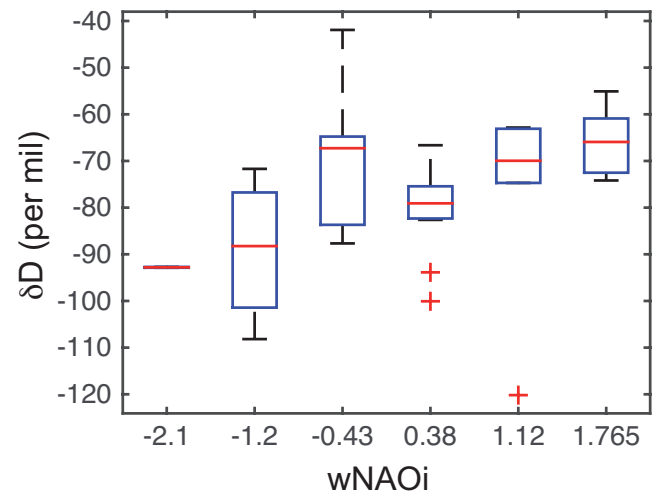

Station 10: Braunschweig 
a)

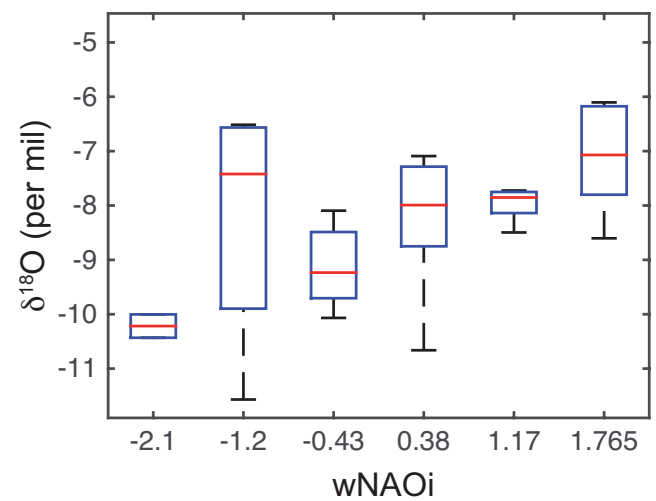

c)

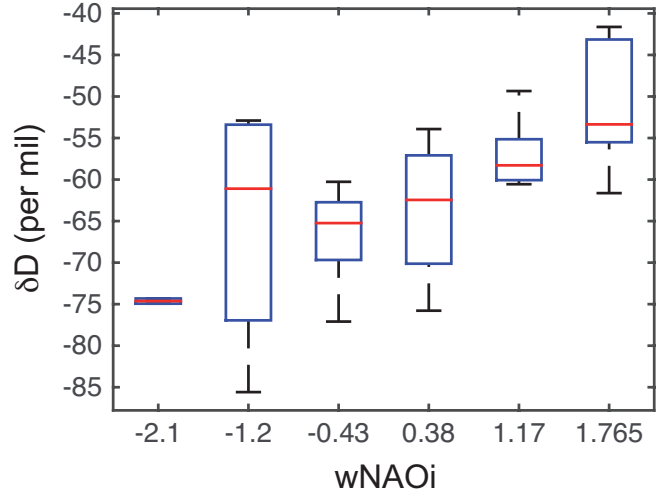

b)

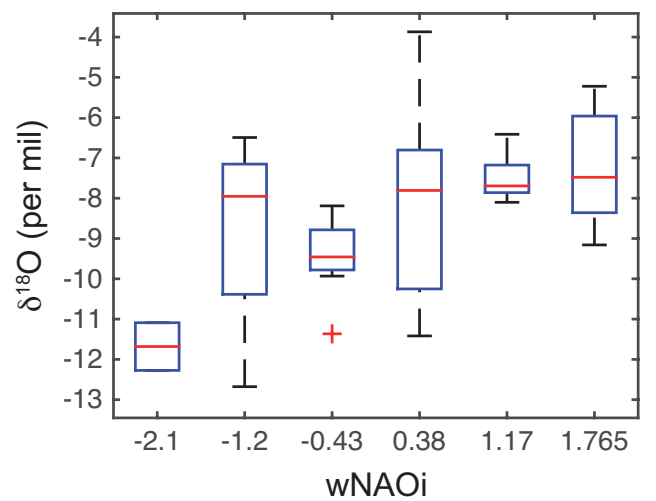

d)

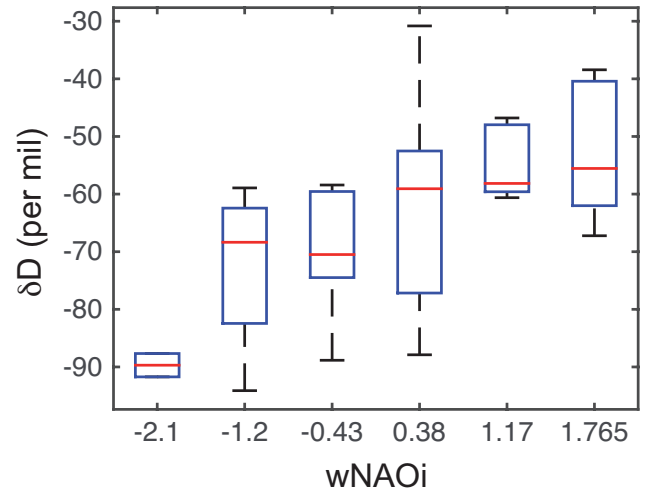

Station 11: Berlin 
a)

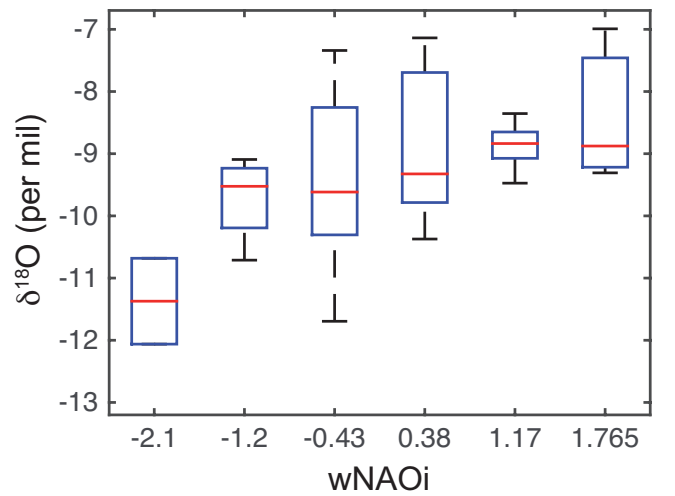

c)

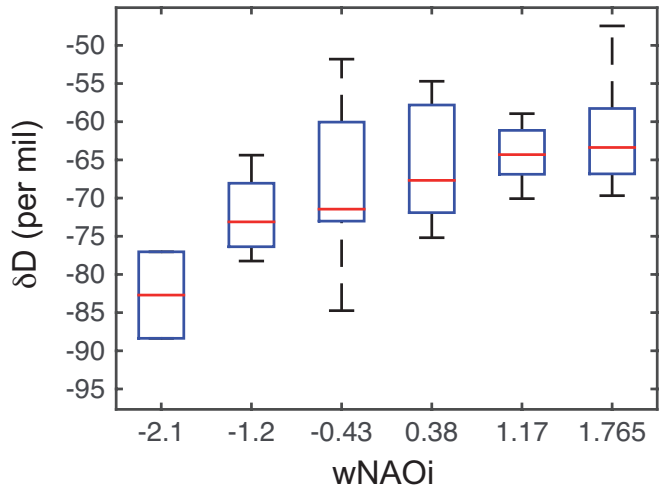

b)

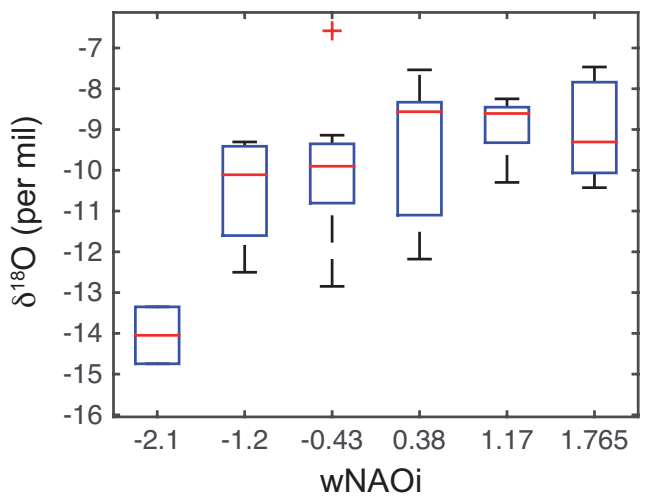

d)

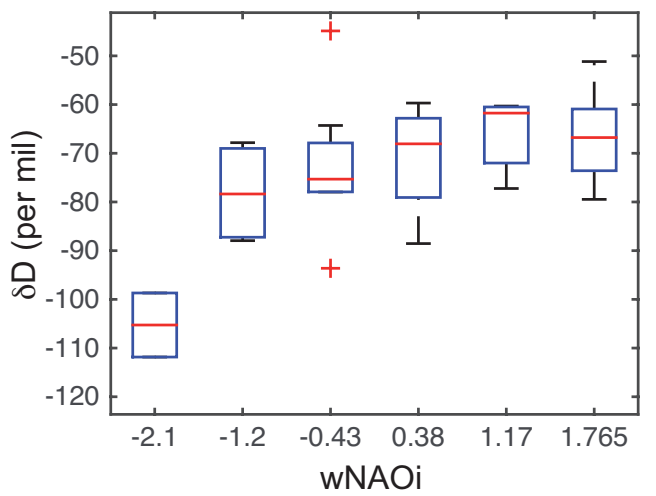

Station 12: Vienna (HOHE WARTE) 
a)

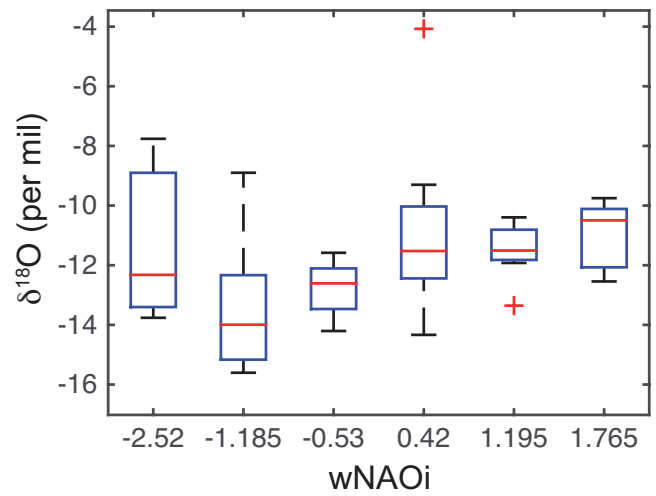

c)

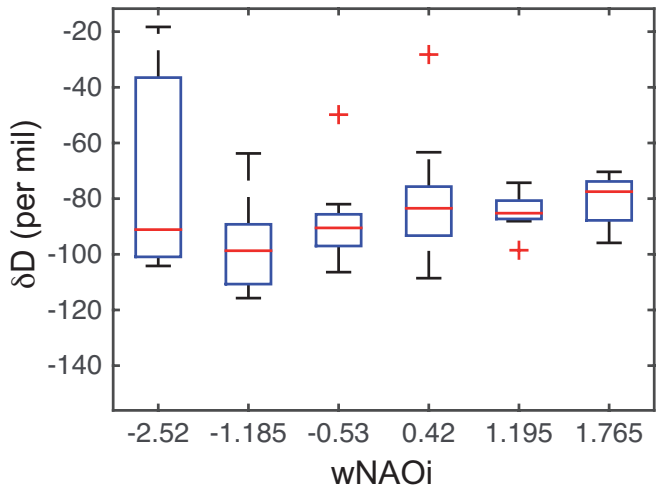

b)

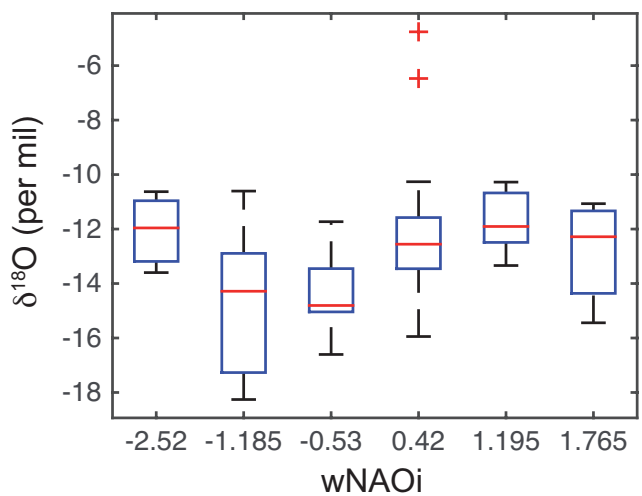

d)

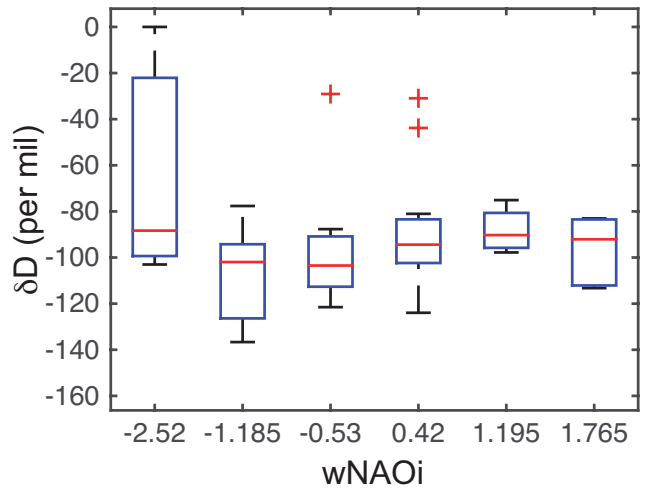

Station 13: Krakow (WOLA JUSTOWSKA) 
a)

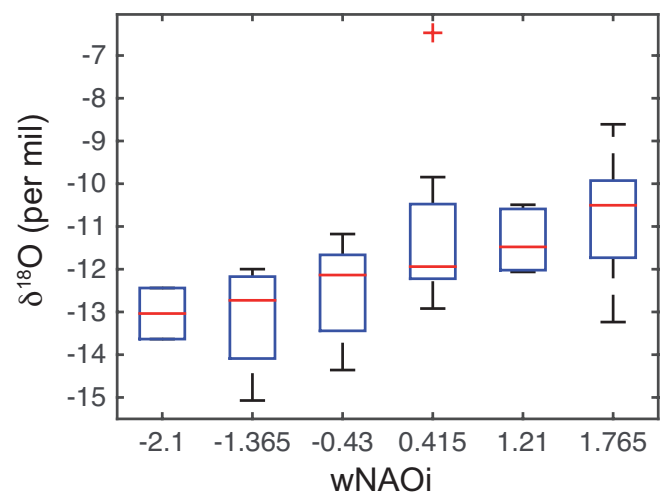

c)

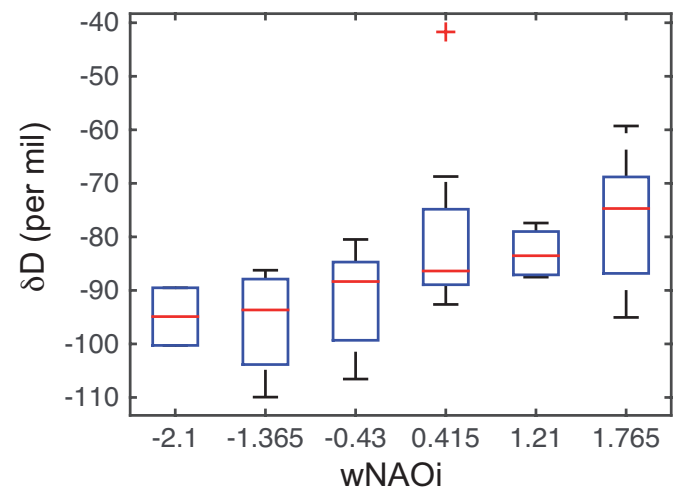

b)

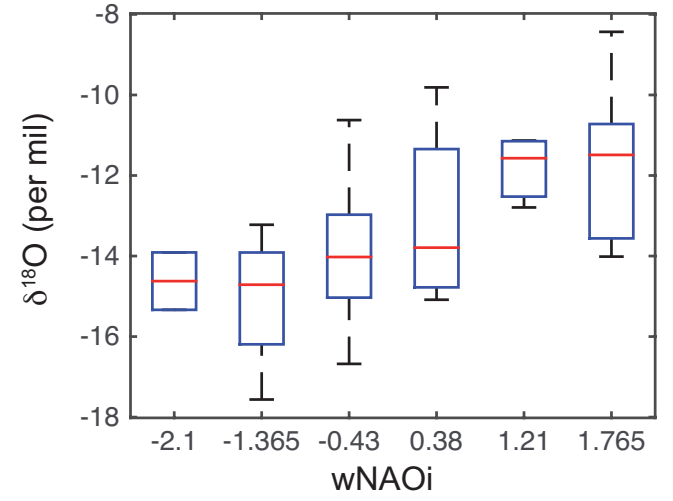

d)

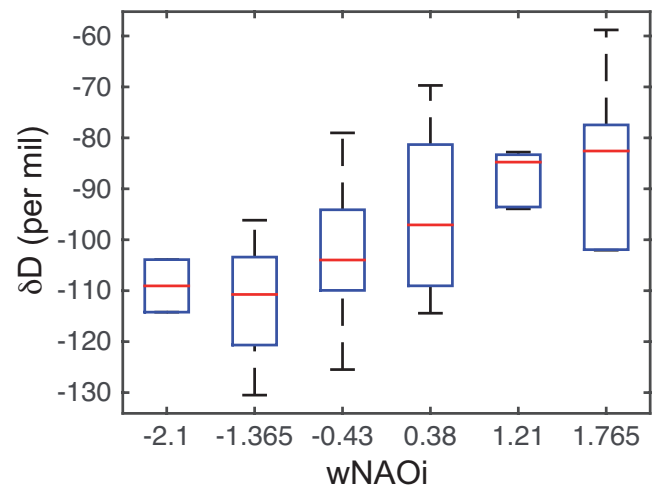

Station 14: Thonon-Les-Bains 
a)

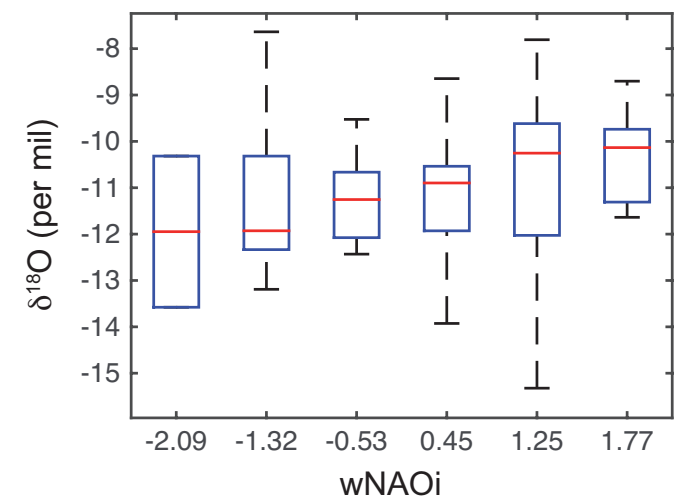

c)

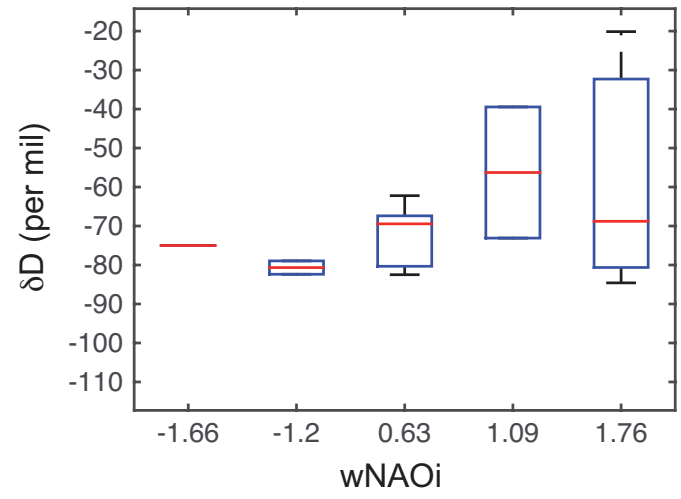

b)

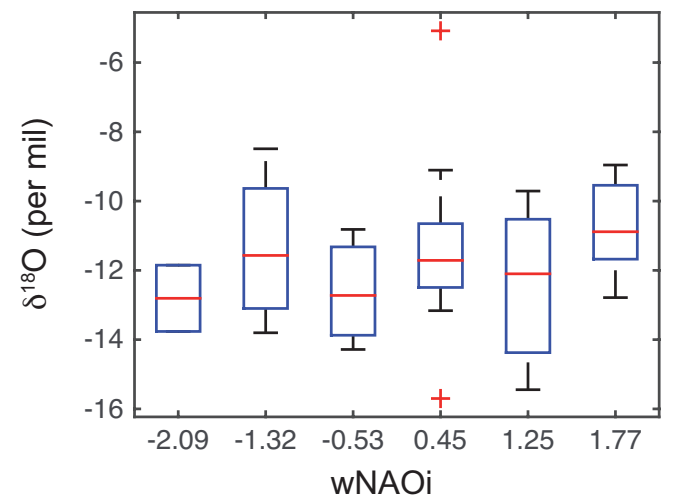

d)

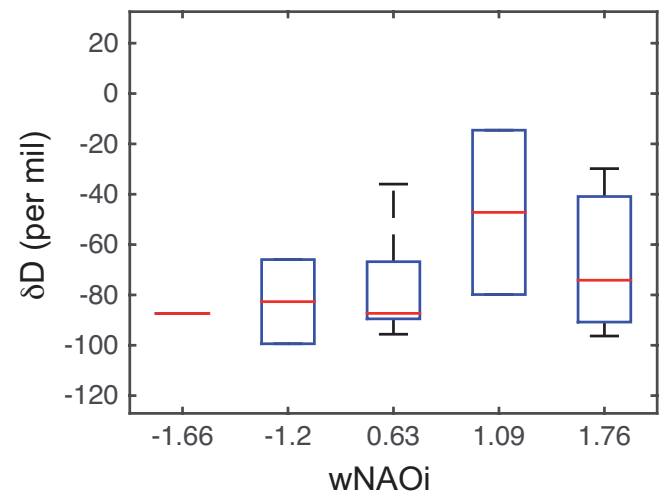

\section{Station 15: Bern}


a)

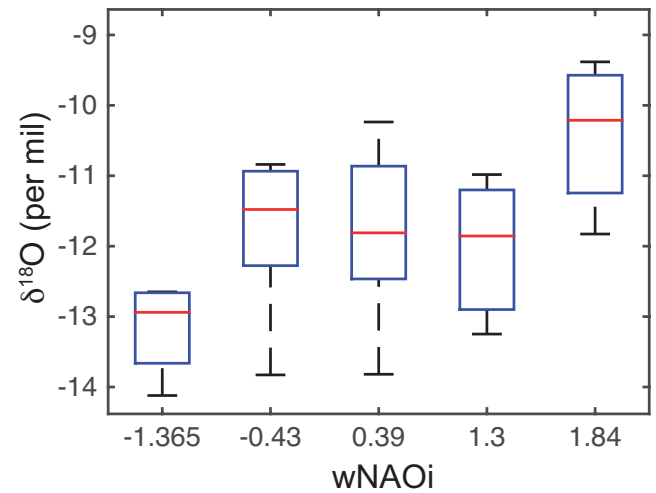

c)

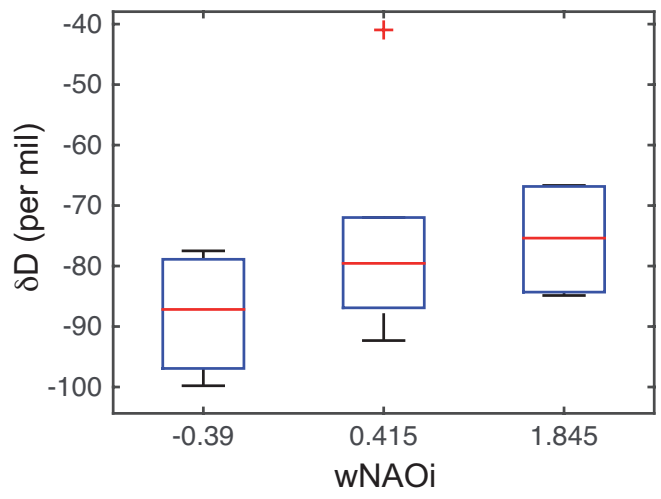

b)

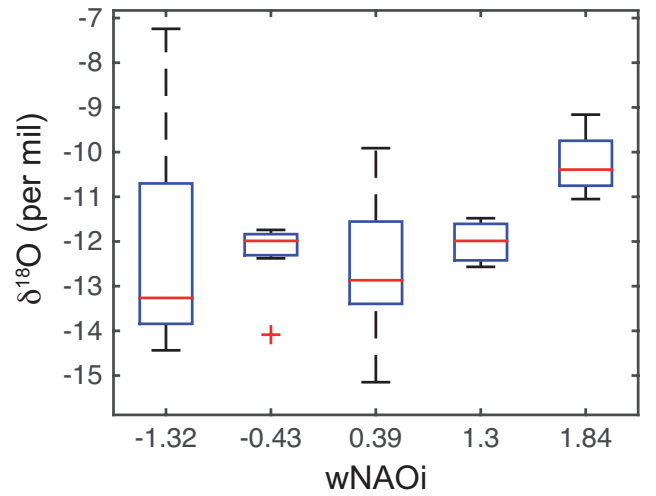

d)

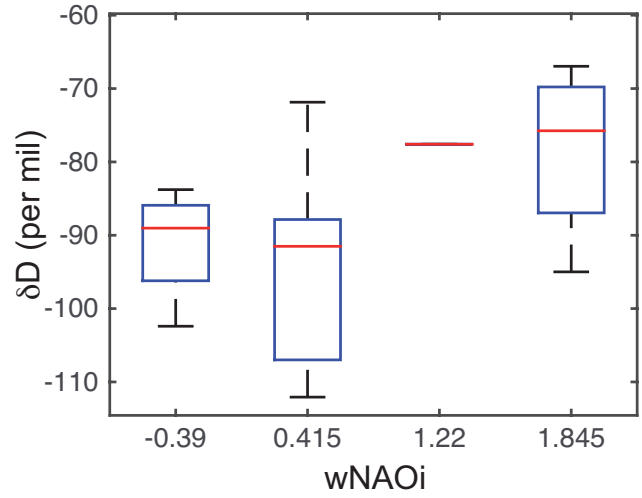

Station 16: Meiringen 
a)

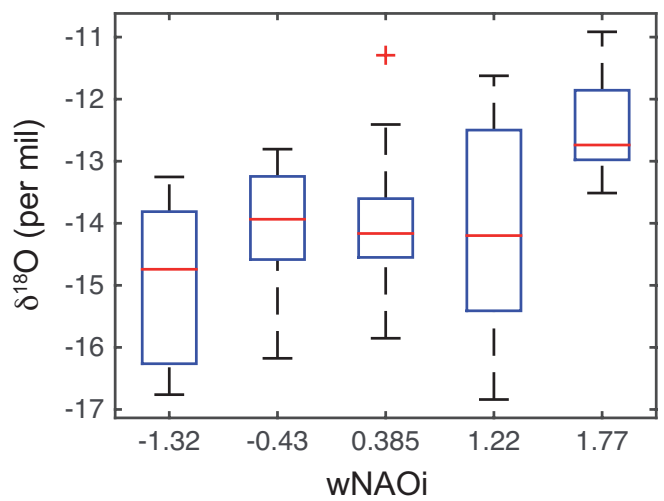

c)

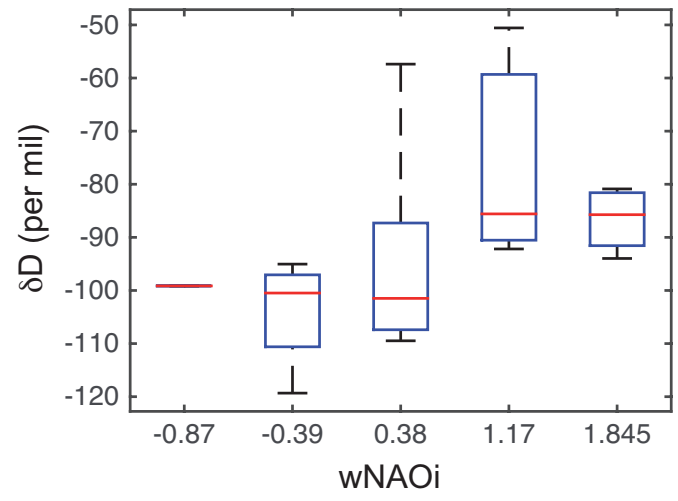

b)

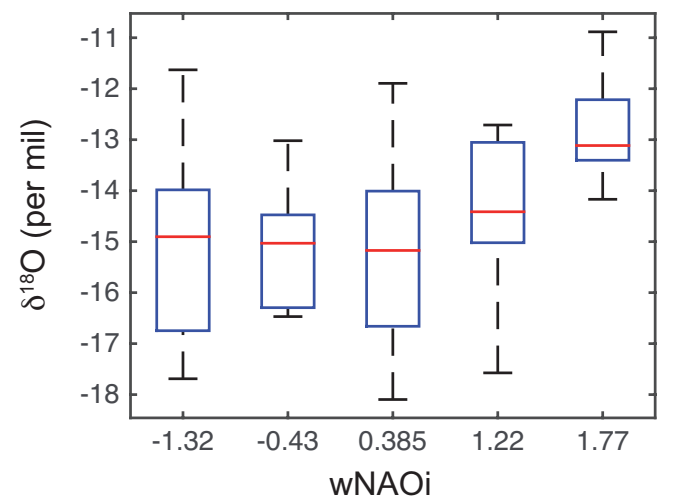

d)

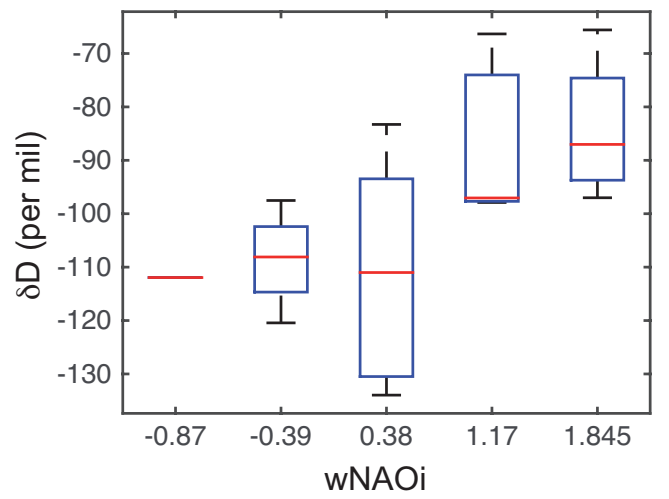

Station 17: Guttannen 
a)

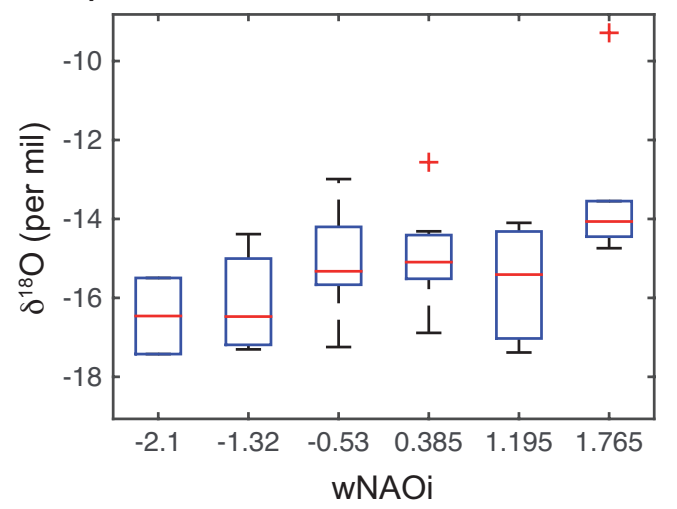

c)

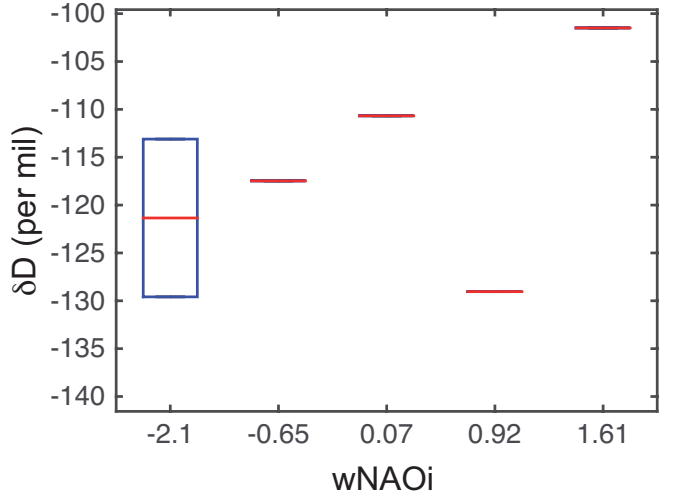

b)

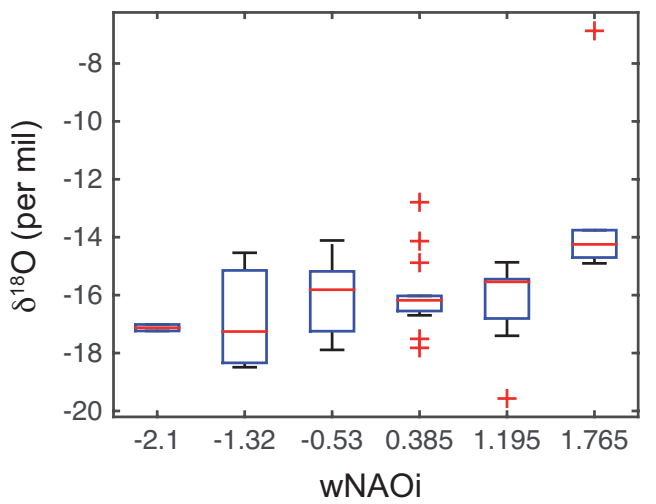

d)

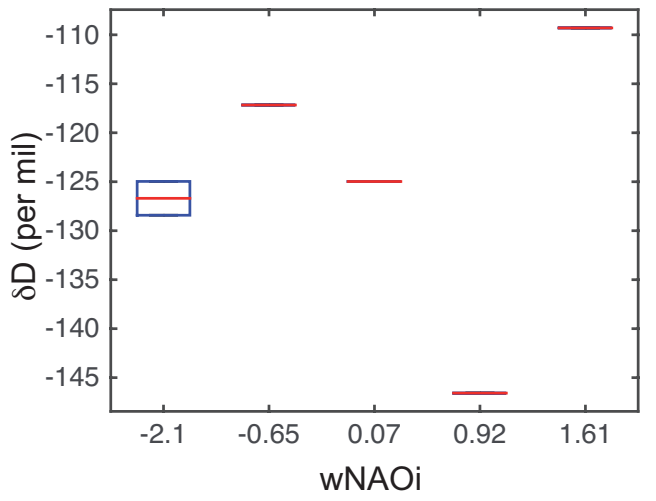

\section{Station 18: Grimsel}


a)

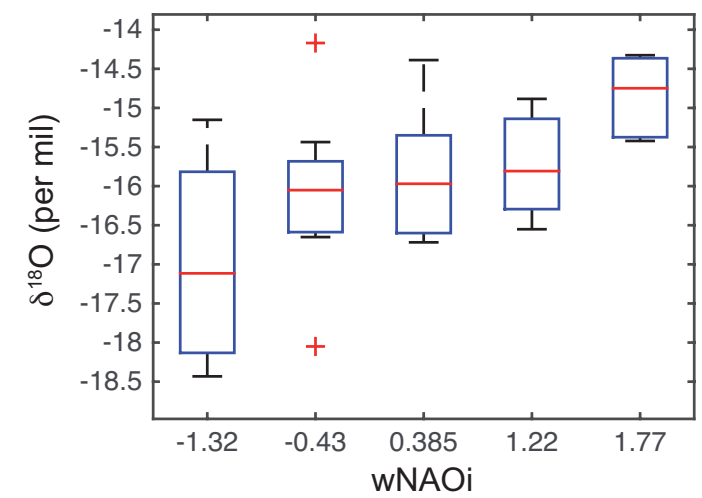

c)

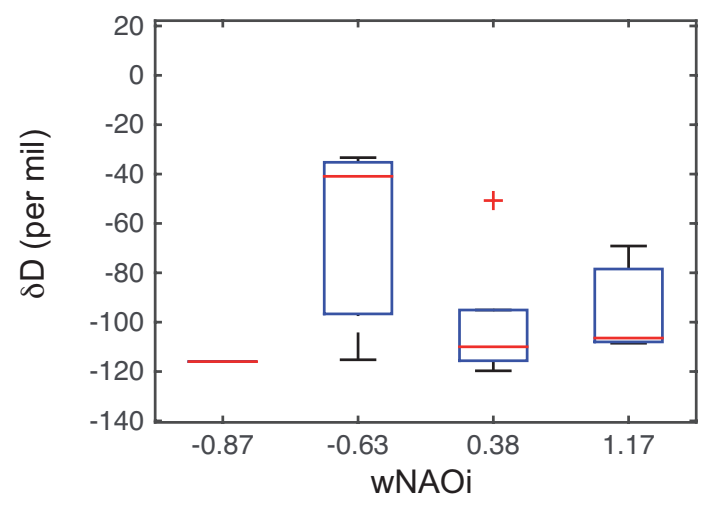

b)

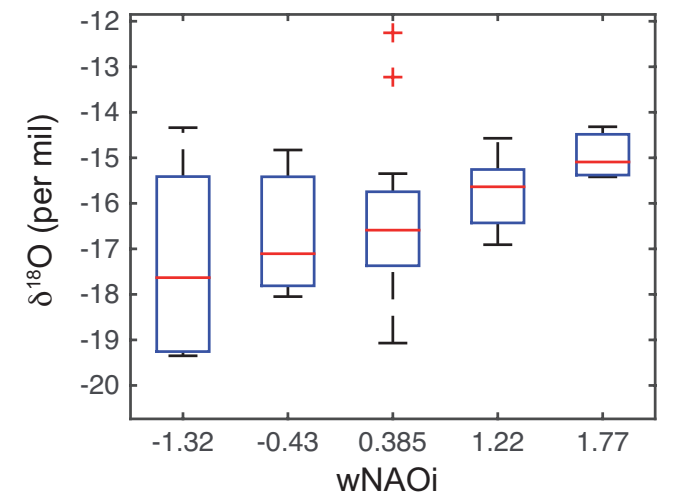

d)

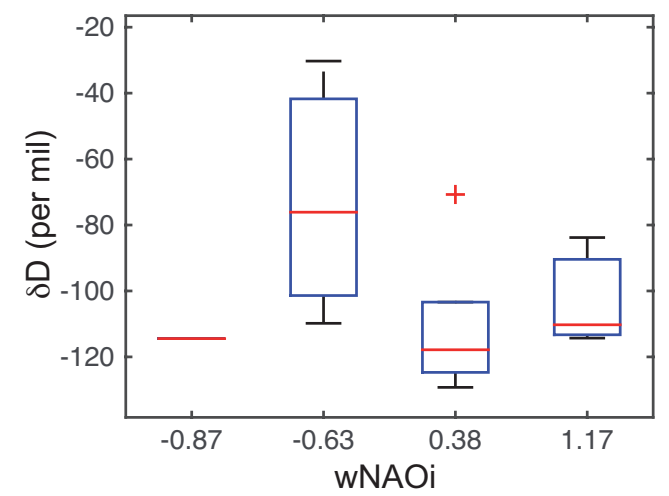

Station 19: Konstanz 
a)

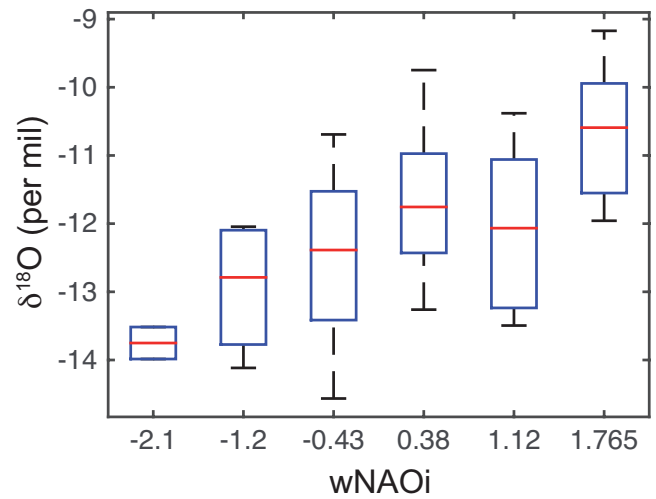

c)

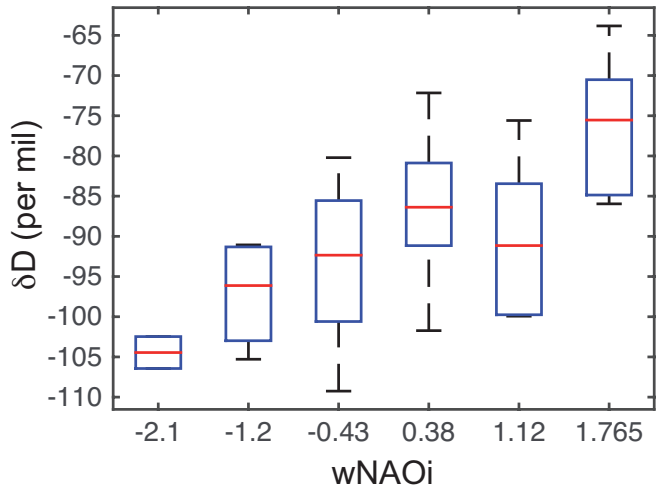

b)

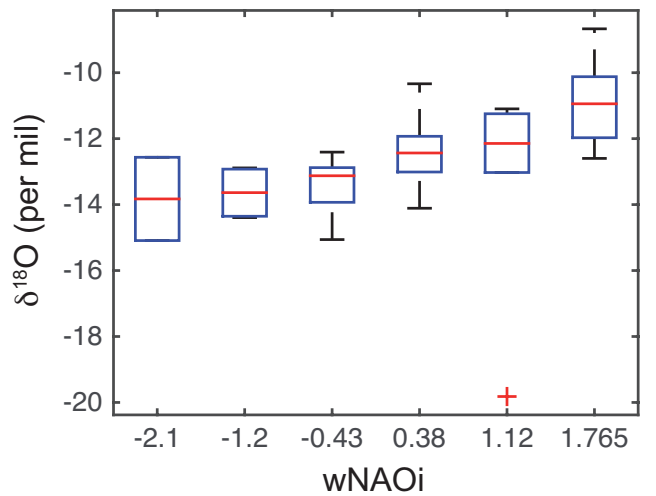

d)

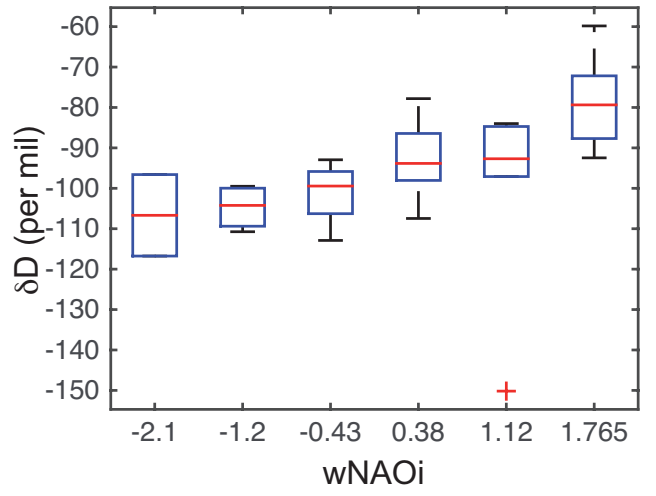

Station 20: Wasserkuppe Rhoen 
a)

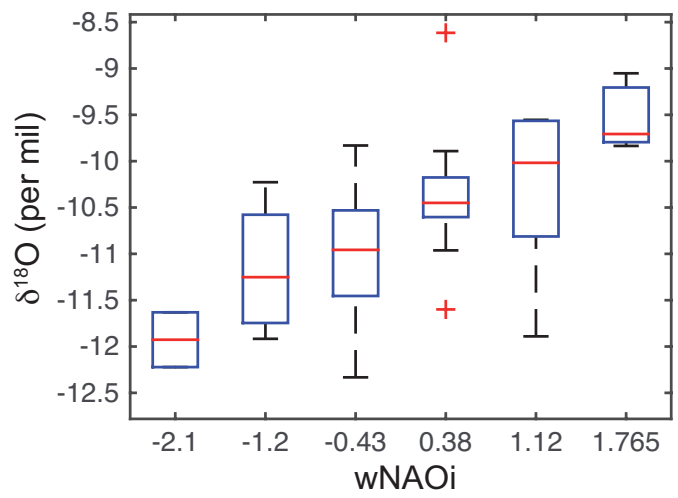

c)

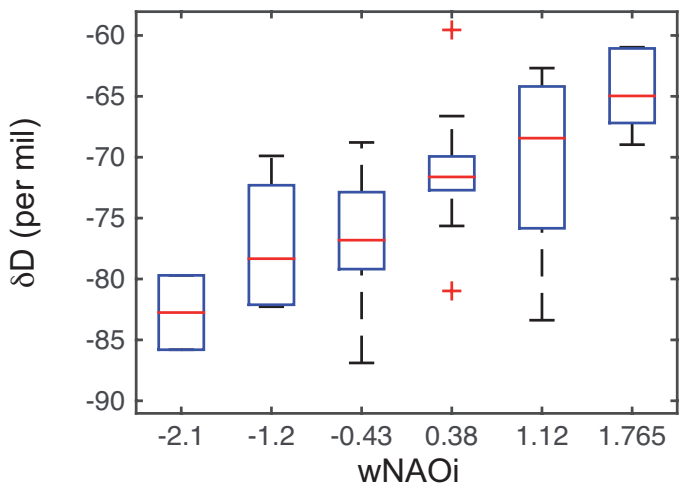

b)

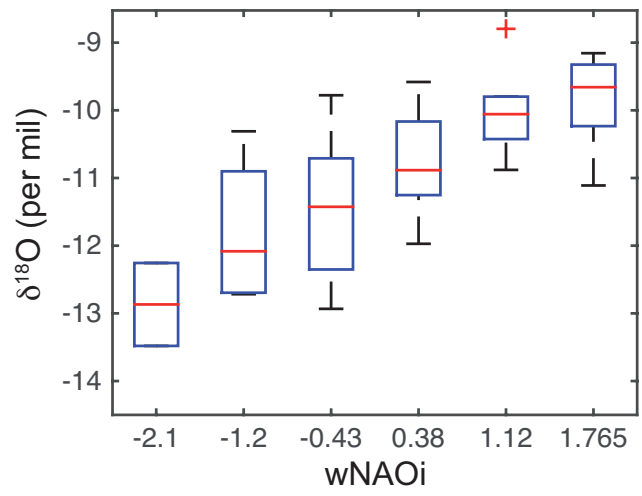

d)

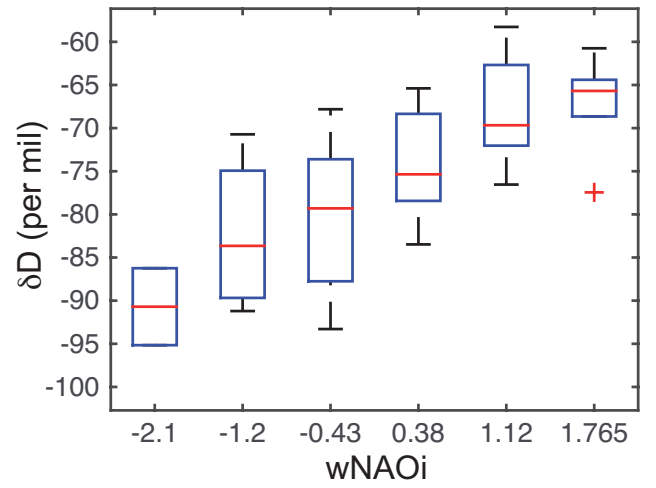

Station 21: Längenfeld 
a)

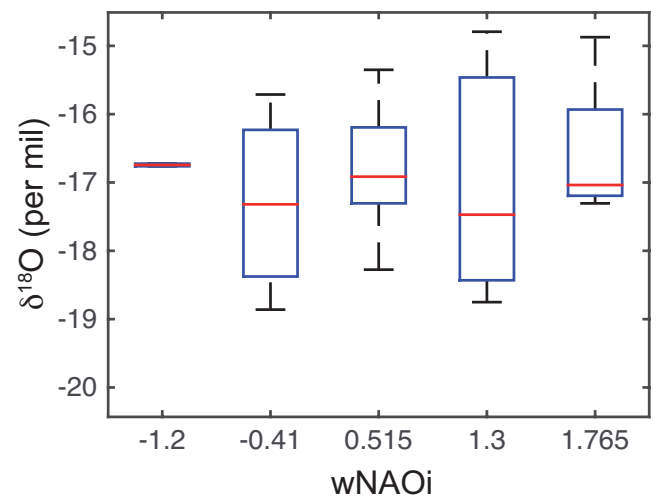

c)

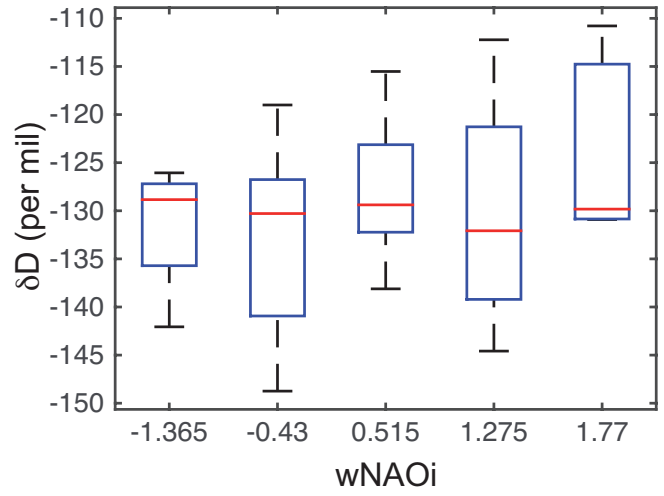

b)

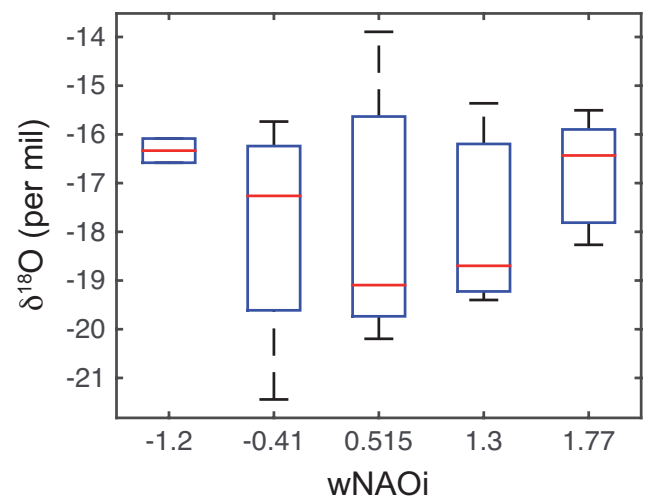

d)

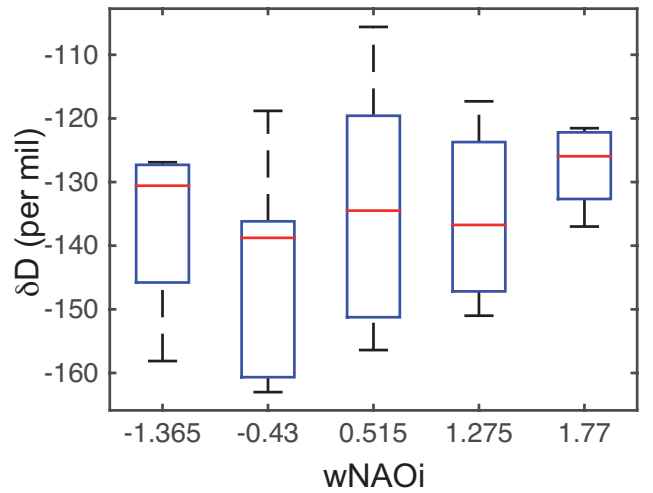

Station 22: Hohenpeissenberg 
a)

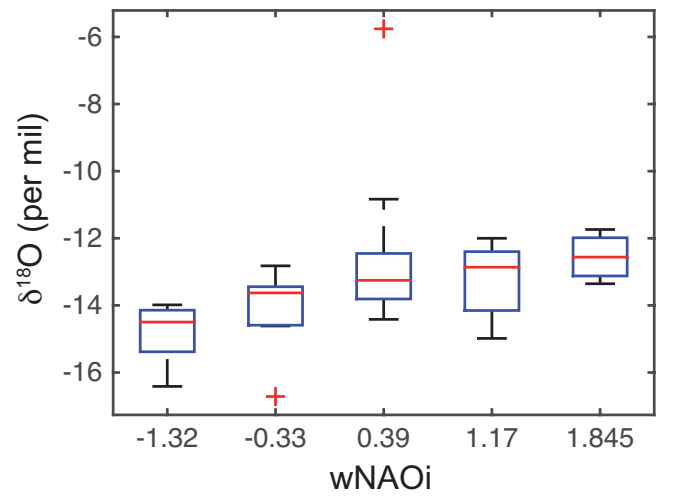

c)

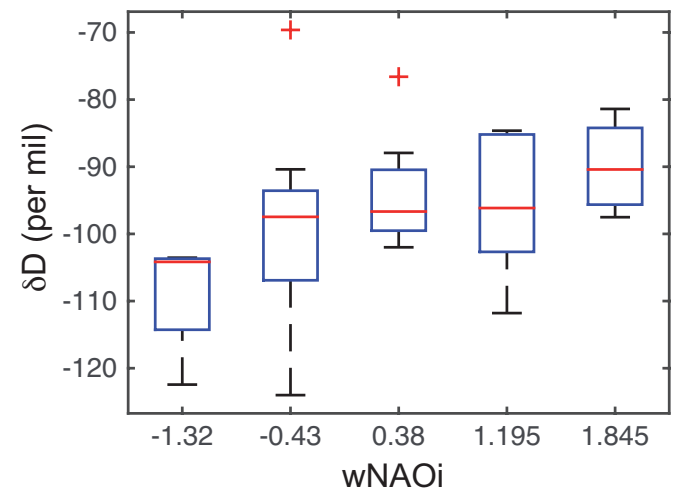

b)

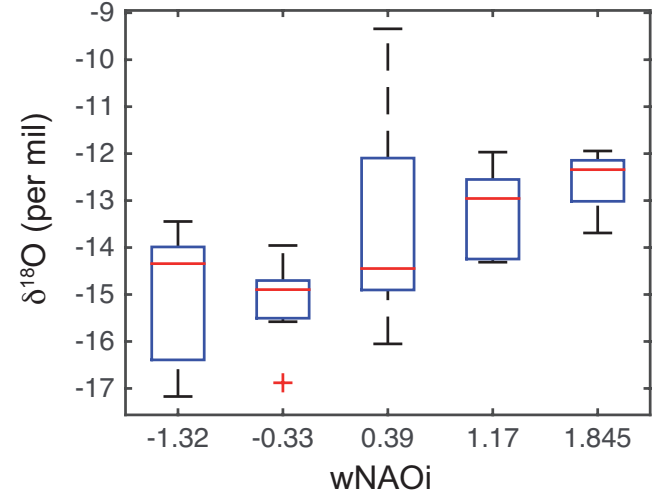

d)

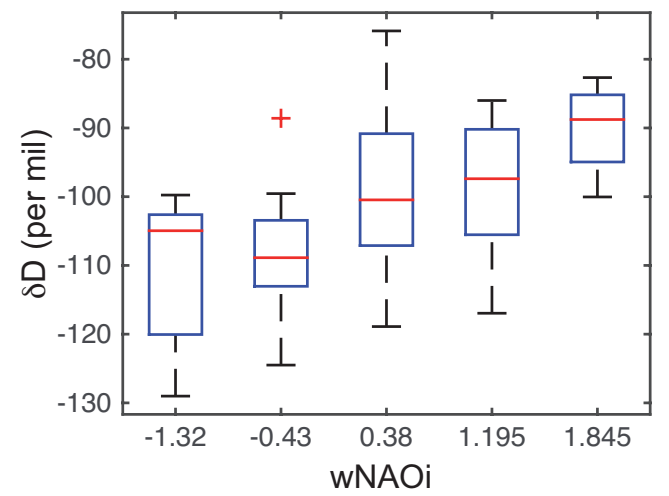

Station 23: Obergurgl 
a)

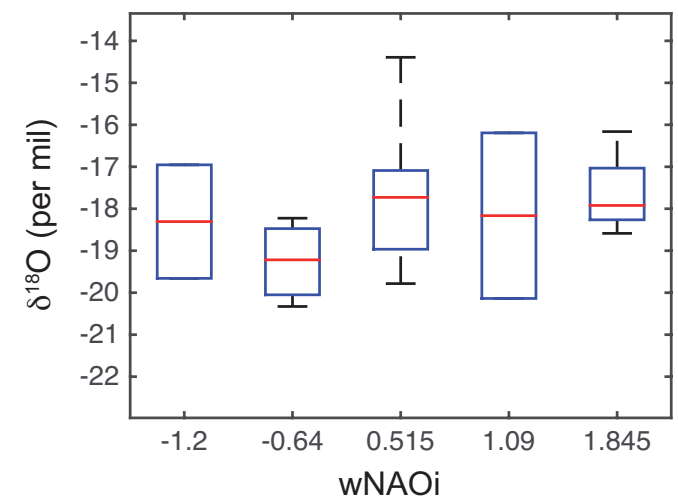

c)

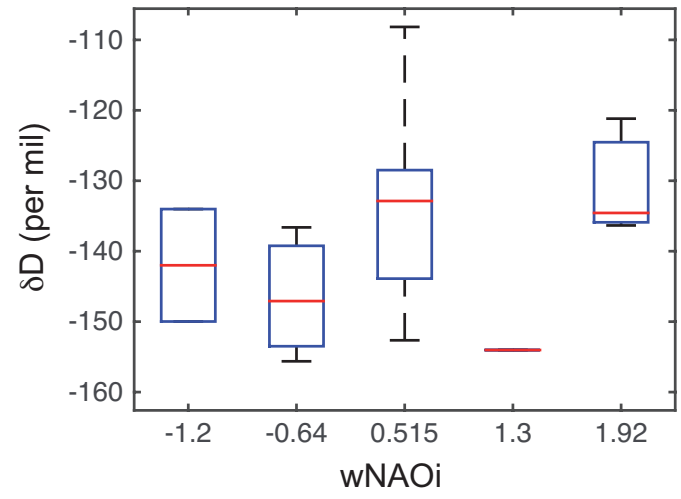

b)

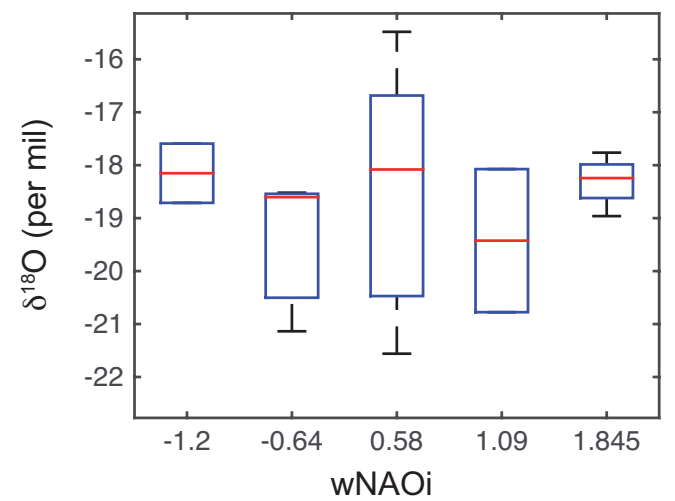

d)

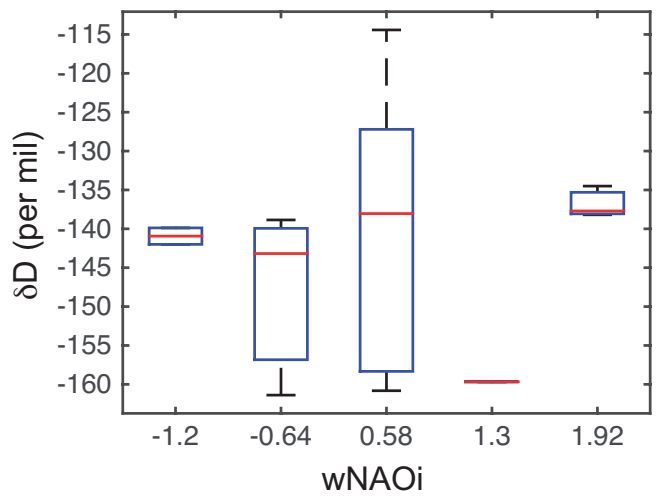

Station 24: Garmisch-Partenkirchen 
a)

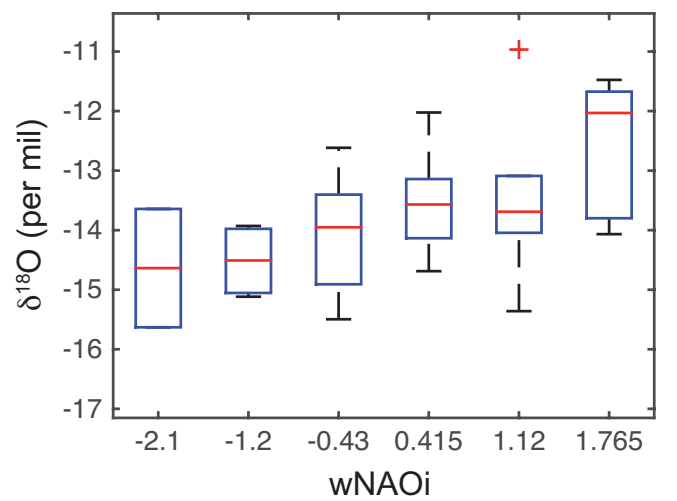

c)

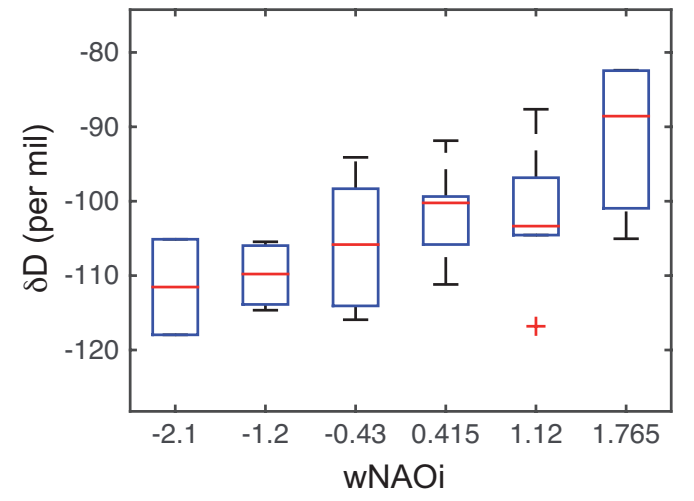

b)

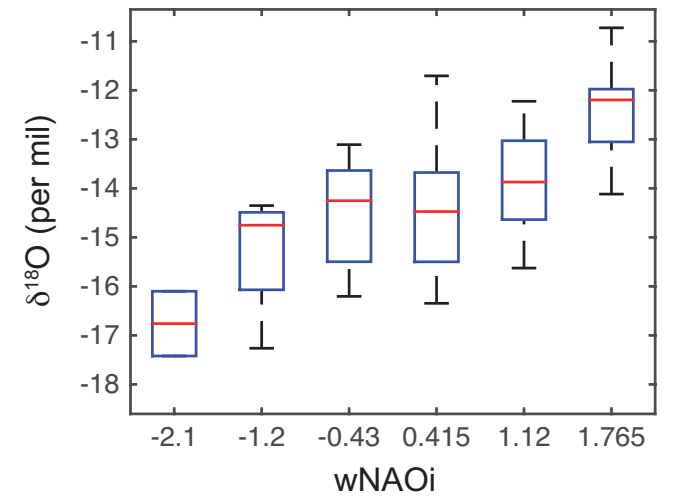

d)

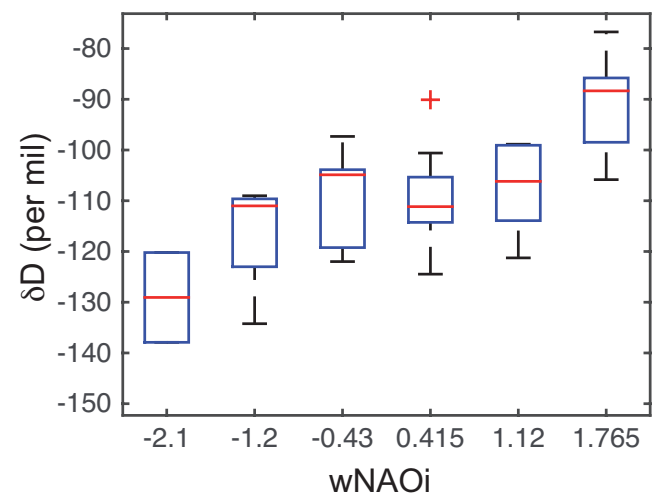

\section{Station 25: Scharnitz}


a)

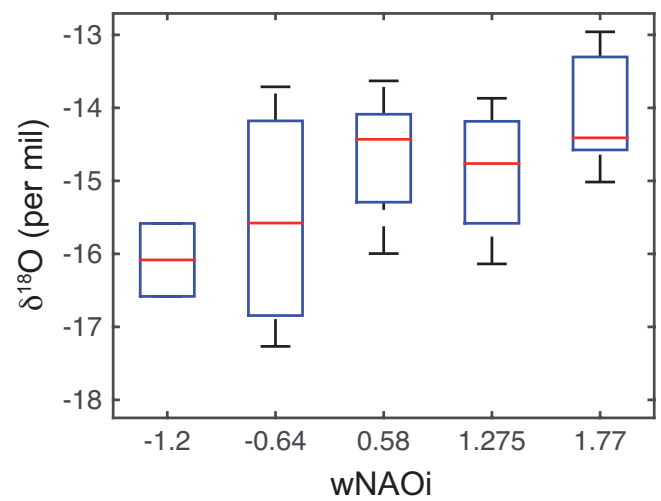

c)

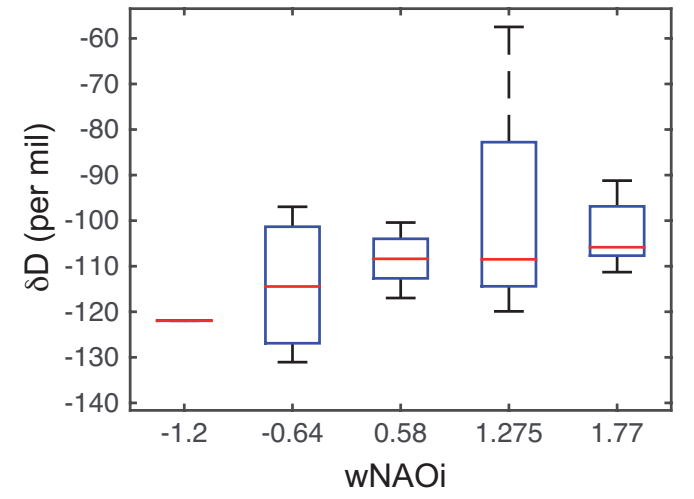

b)

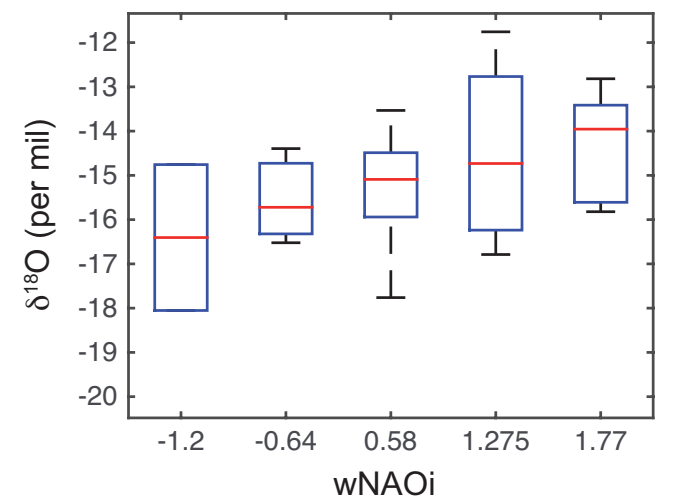

d)

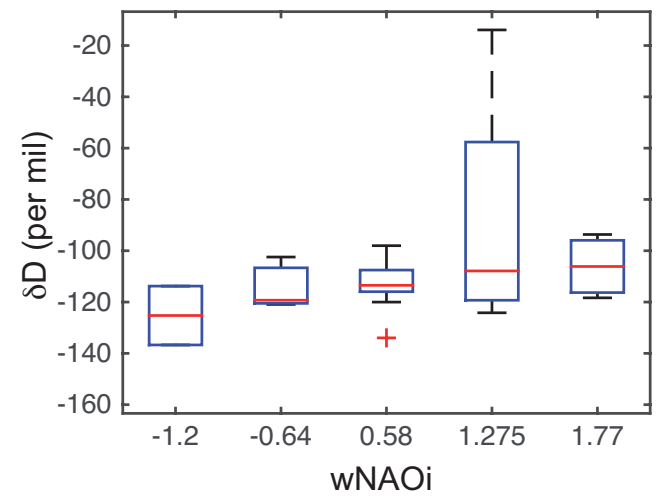

Station 26: Neuherberg 
a)

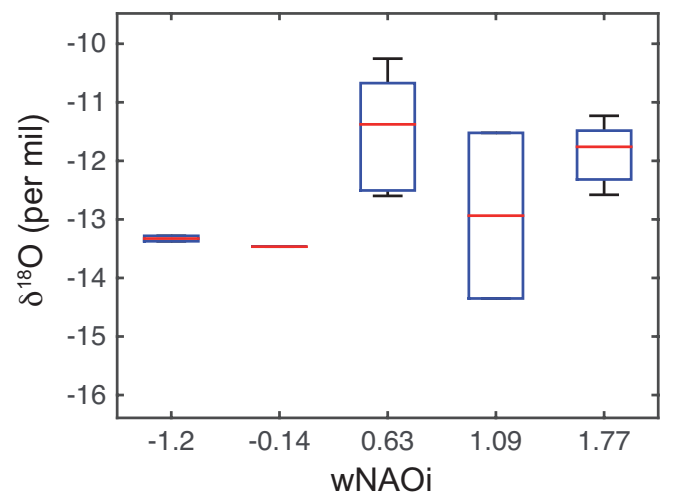

c)

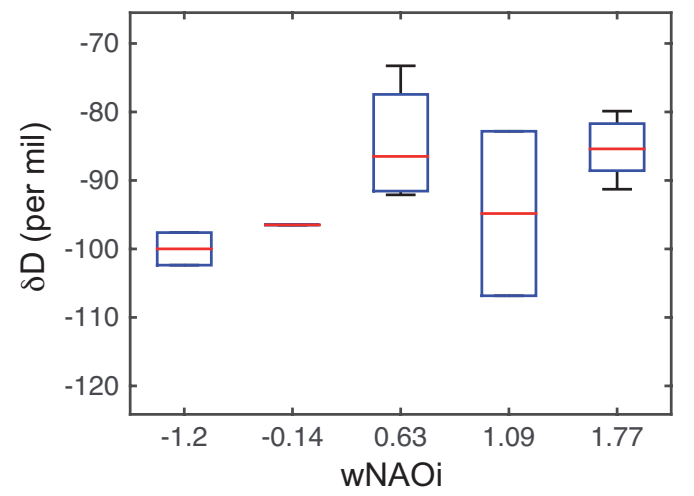

b)

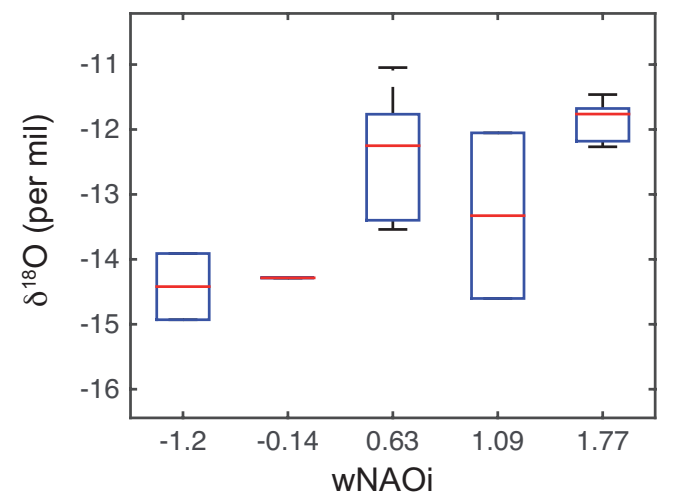

d)

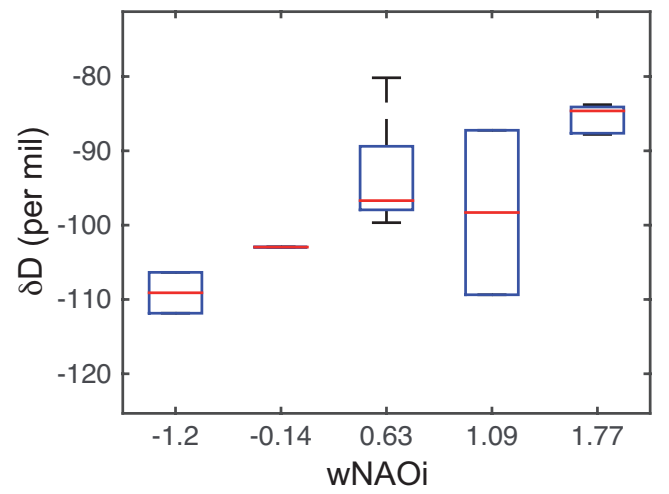

Station 27: Pastscherkofel 
a)

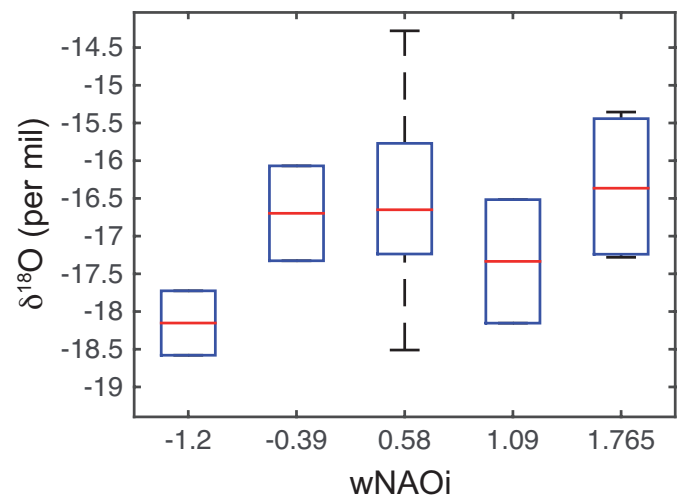

c)

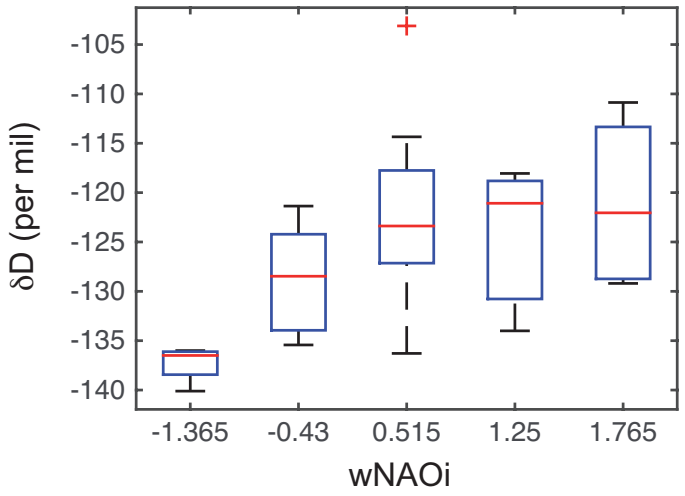

b)

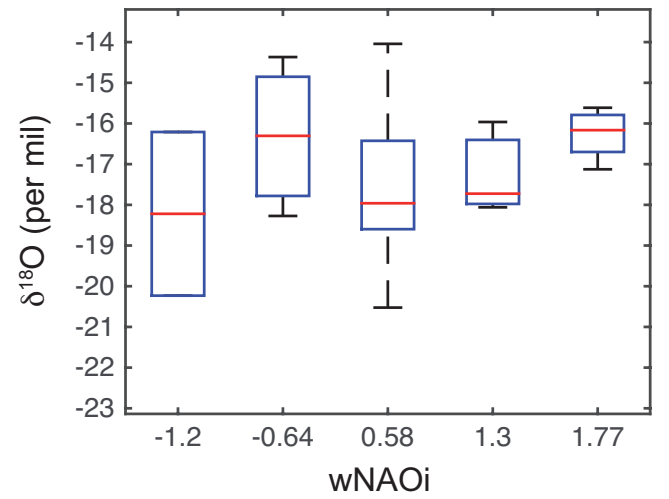

d)

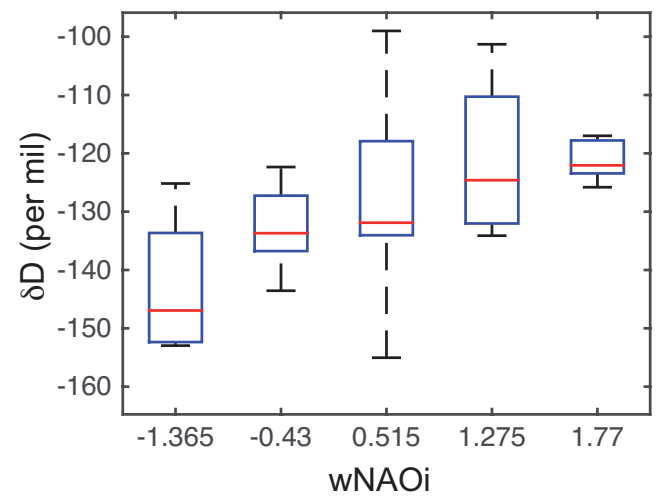

Station 28: Hof-Hohensaas 
a)

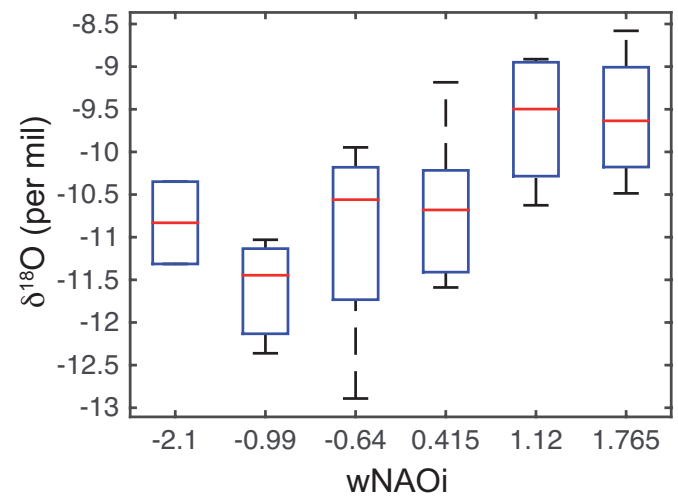

c)

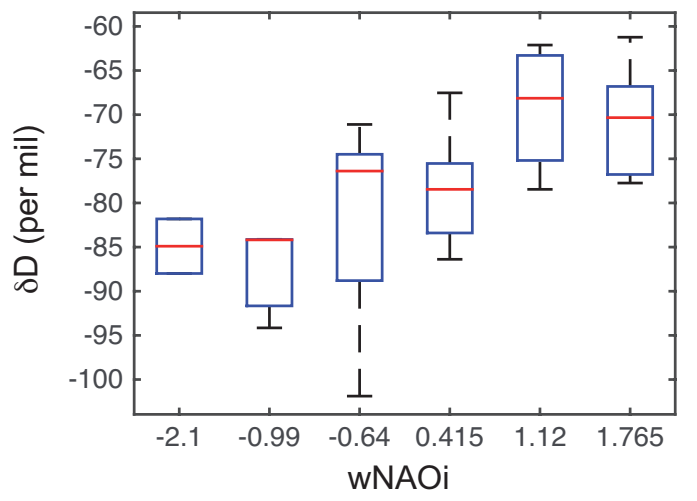

b)

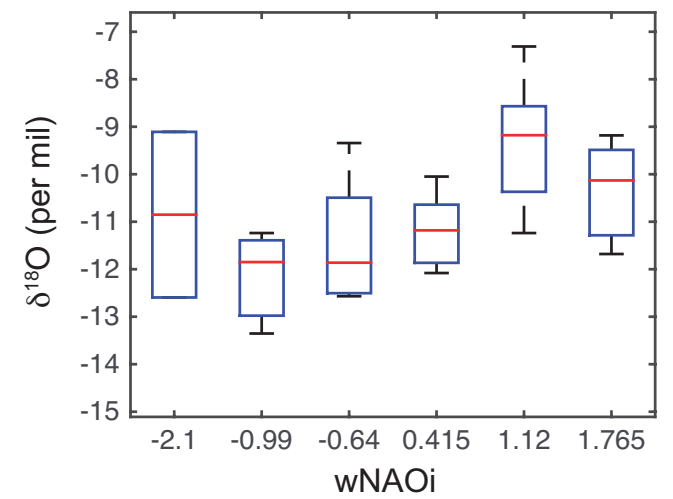

d)

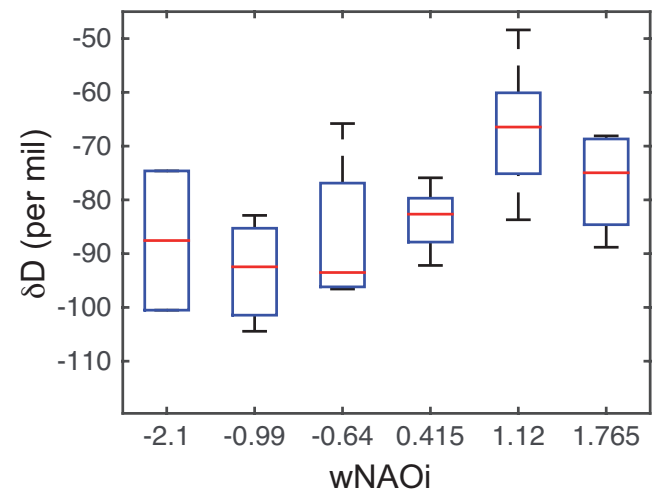

Station 29: Regensburg 
a)

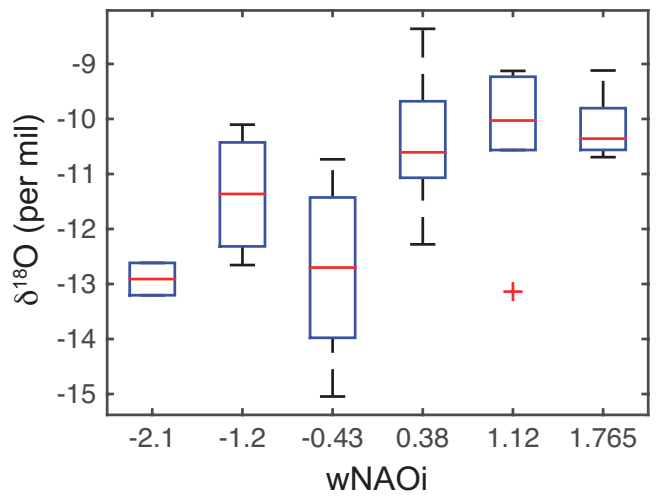

c)

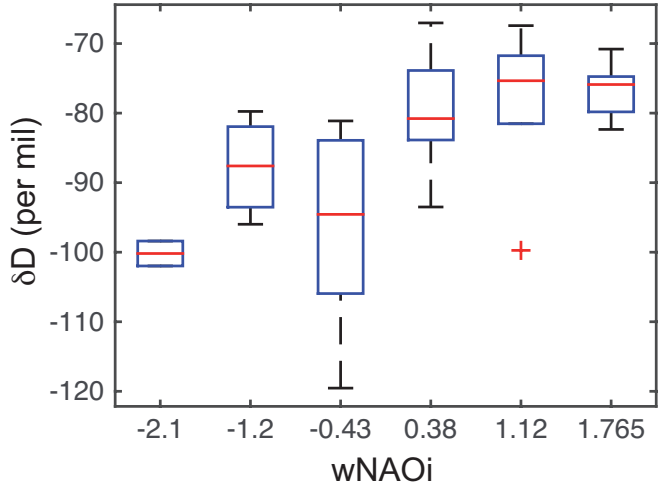

b)

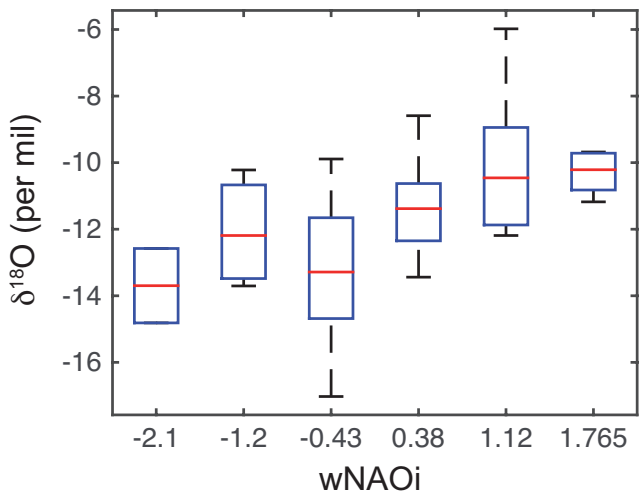

d)

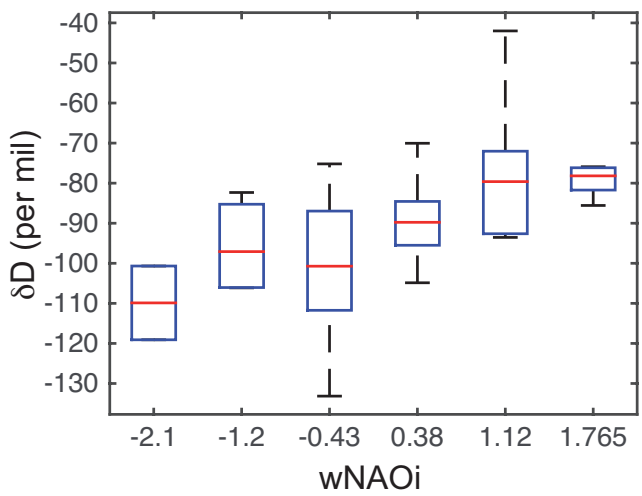

Station 30: Böckstein 
a)

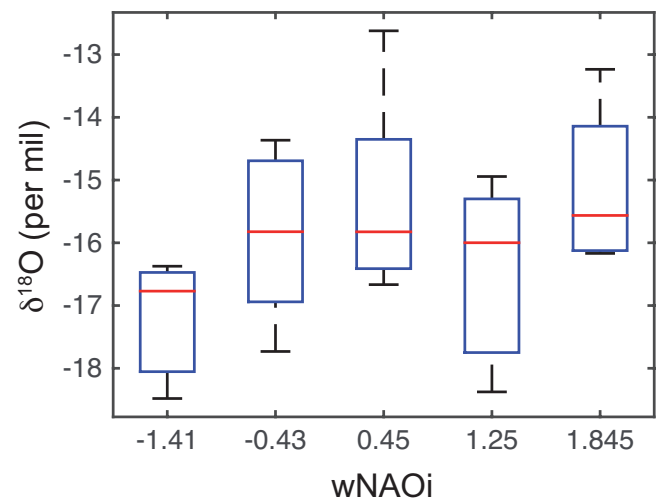

c)

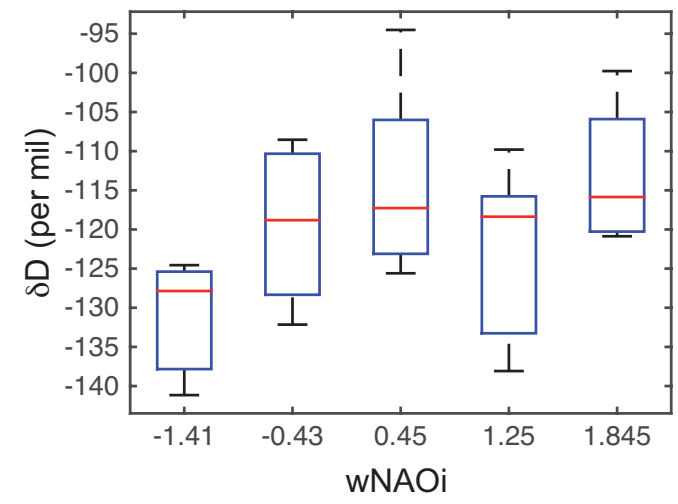

b)

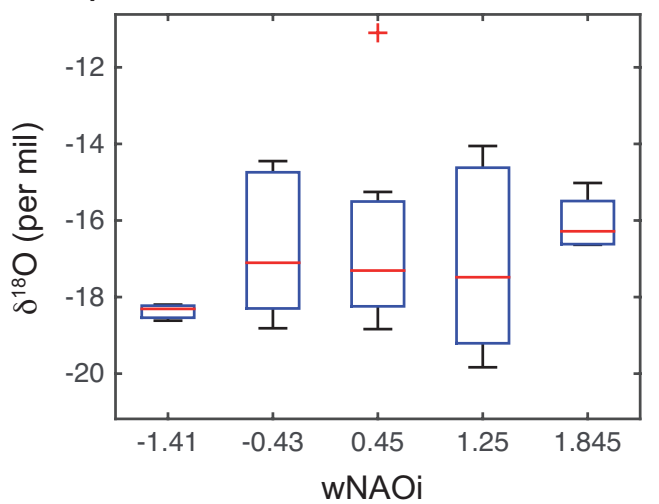

d)

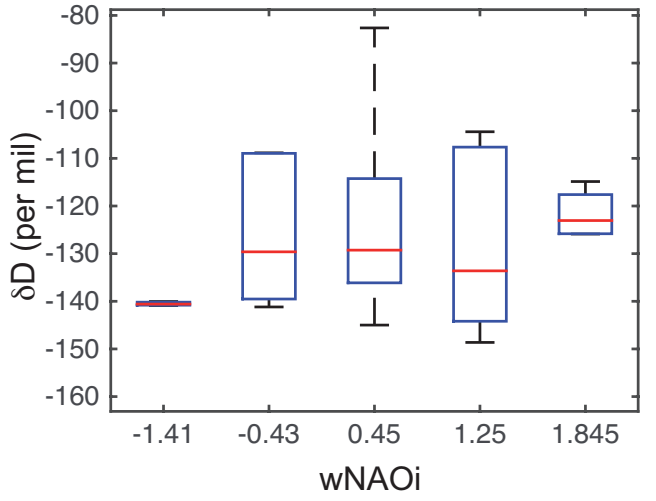

Station 31: St Peter 
a)

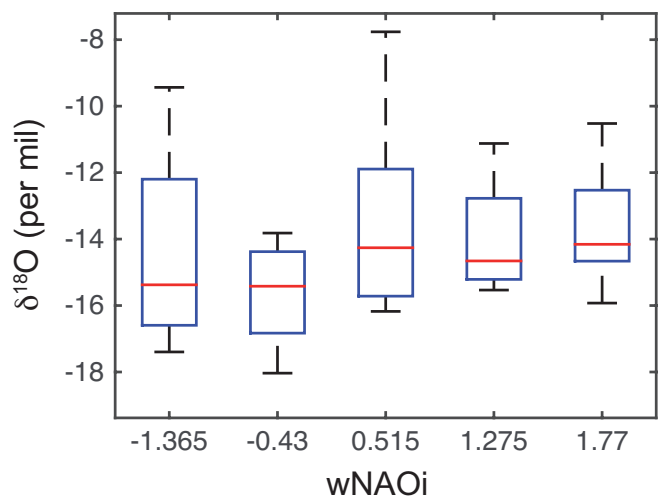

c)

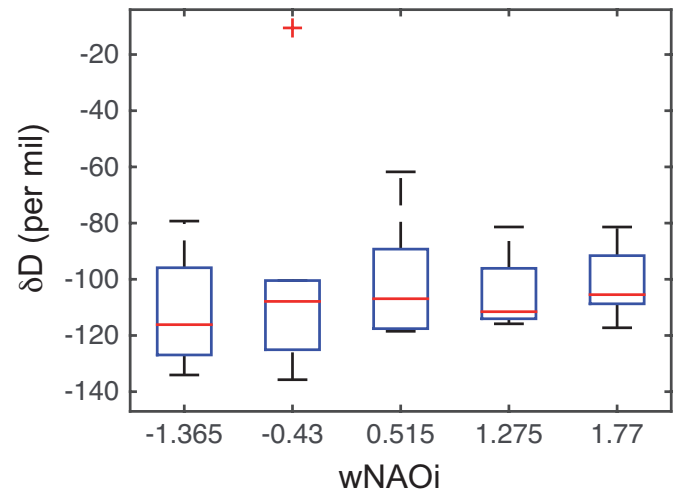

b)

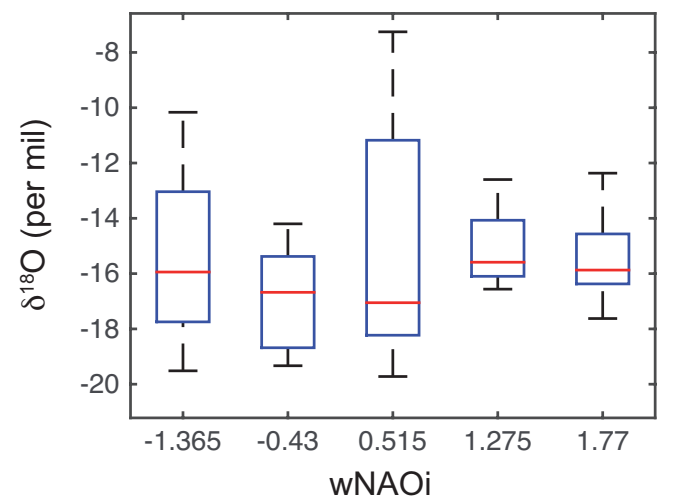

d)

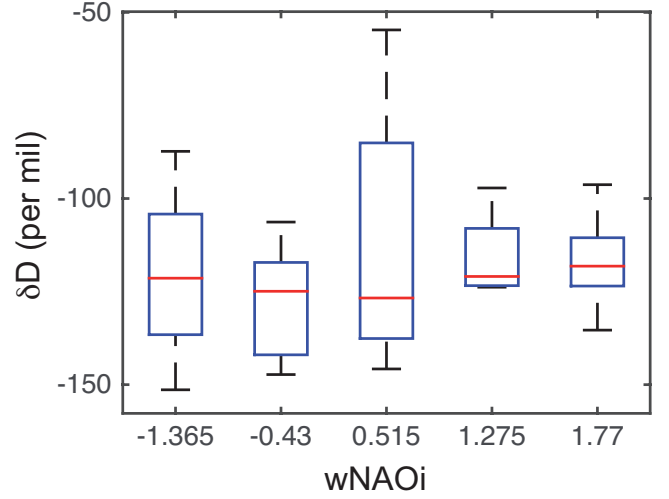

Station 32: Villacher Alpe 
a)

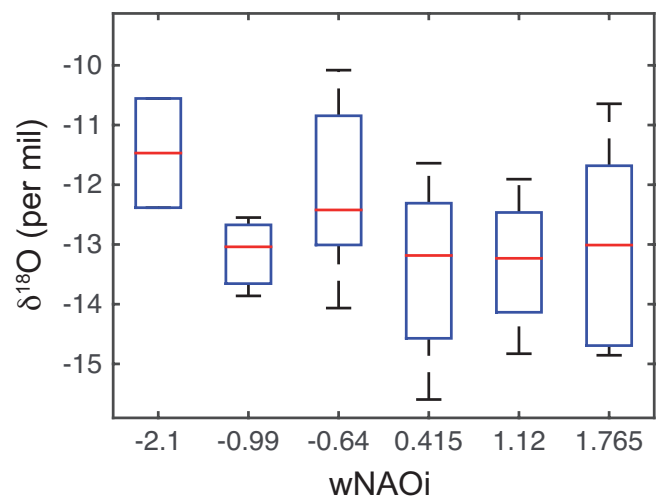

c)

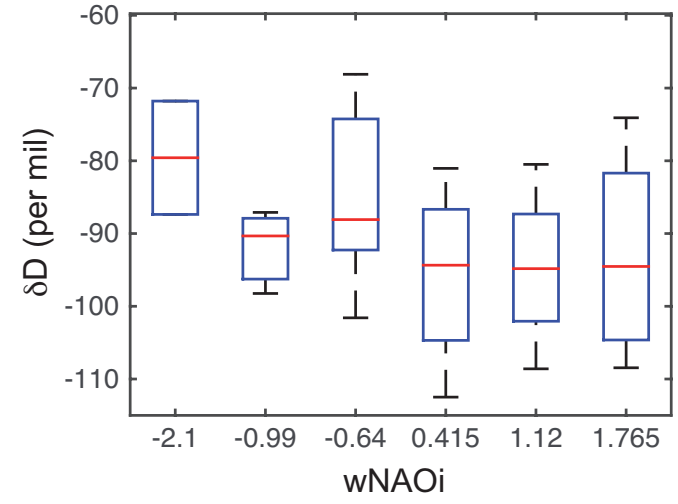

b)

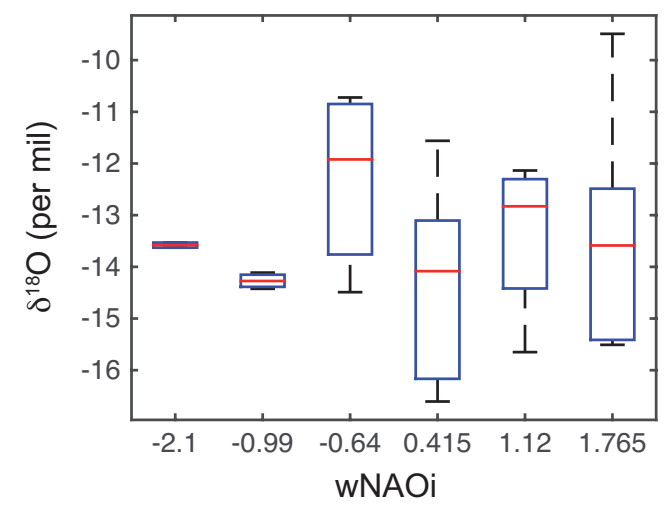

d)

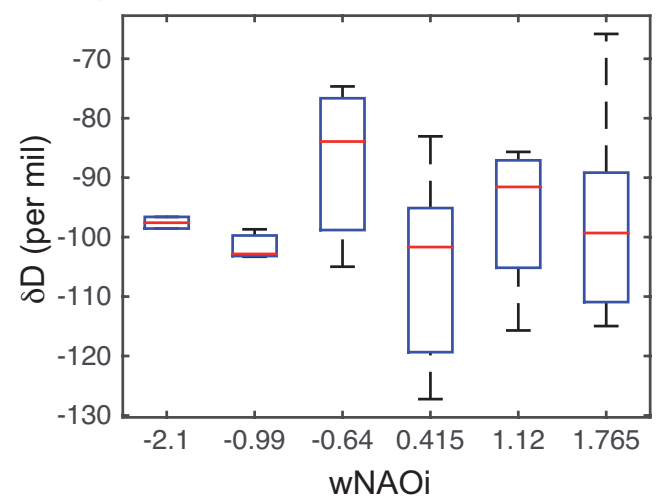

Station 33: Graz Universität 
a)

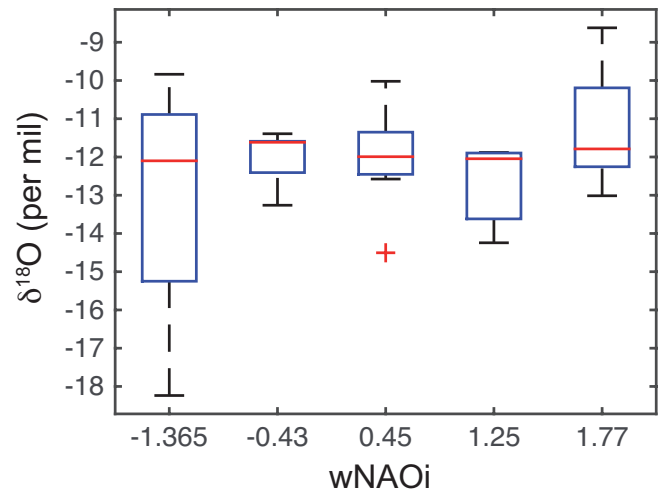

c)

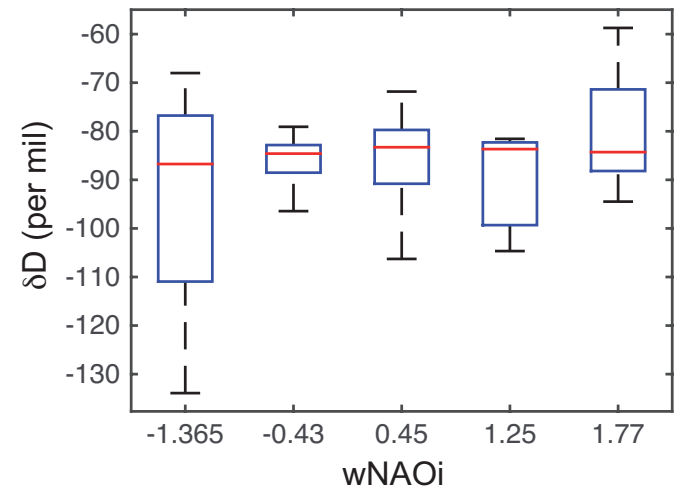

b)

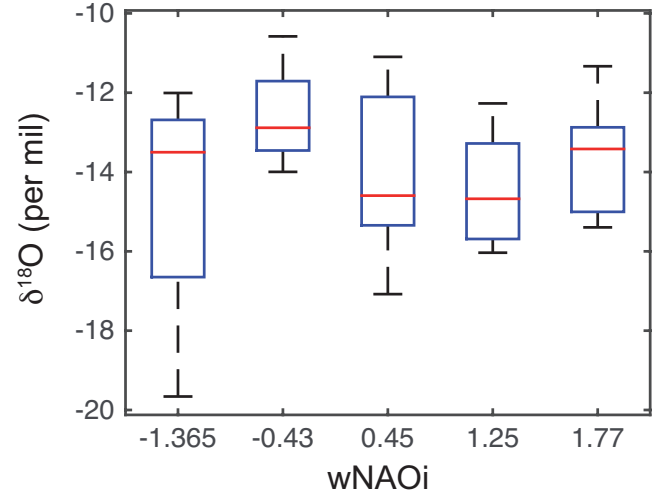

d)

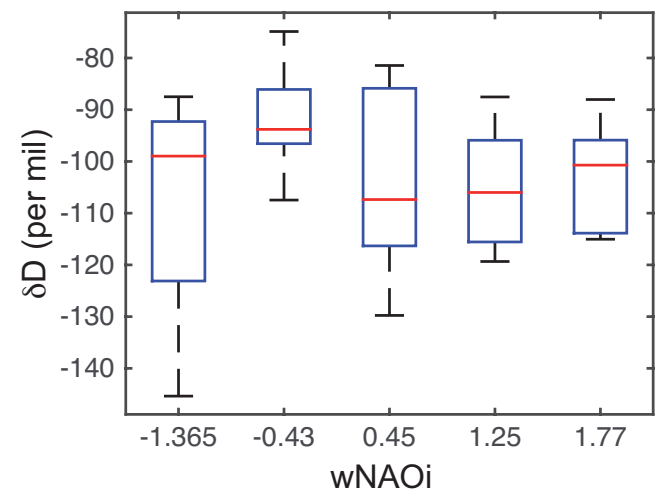

Station 34: Avignon 
a)

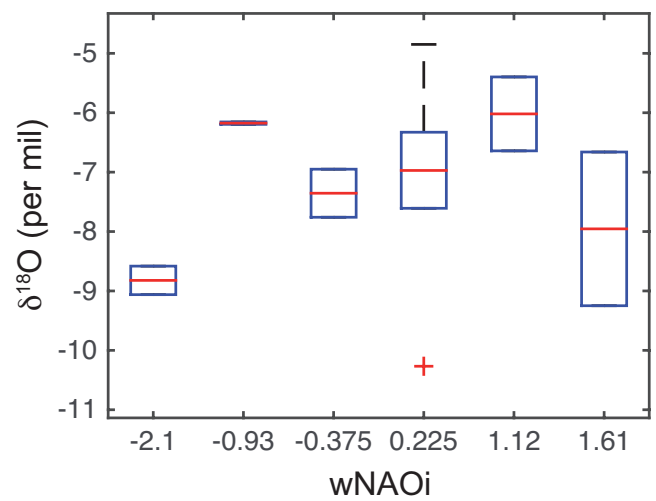

c)

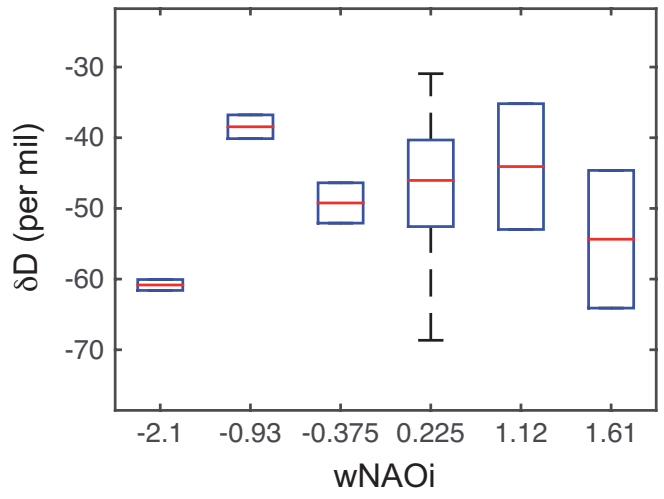

b)

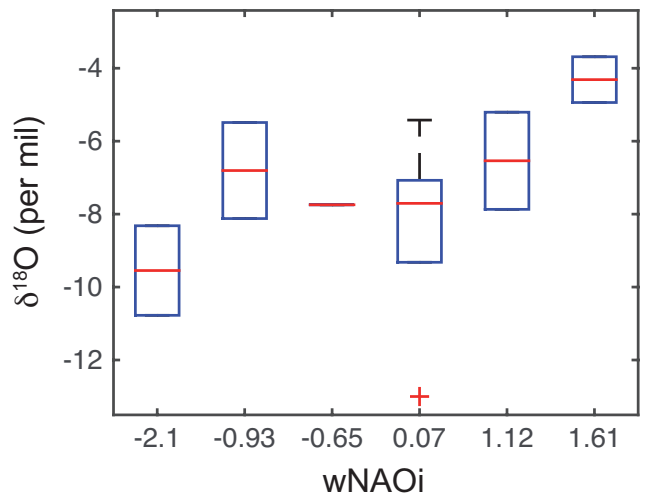

d)

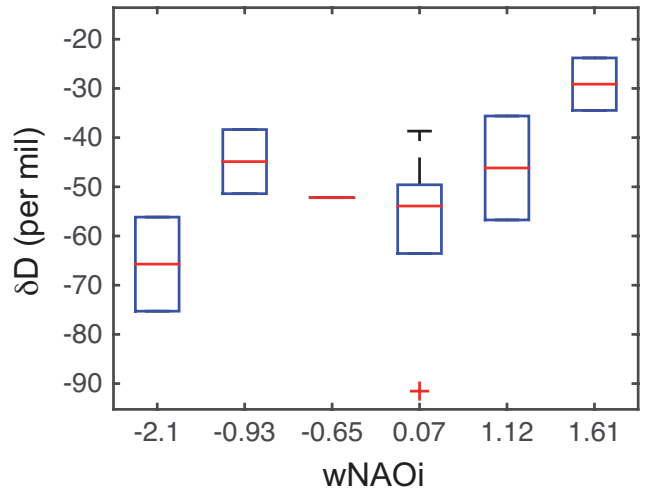

Station 35: Locarno 
a)

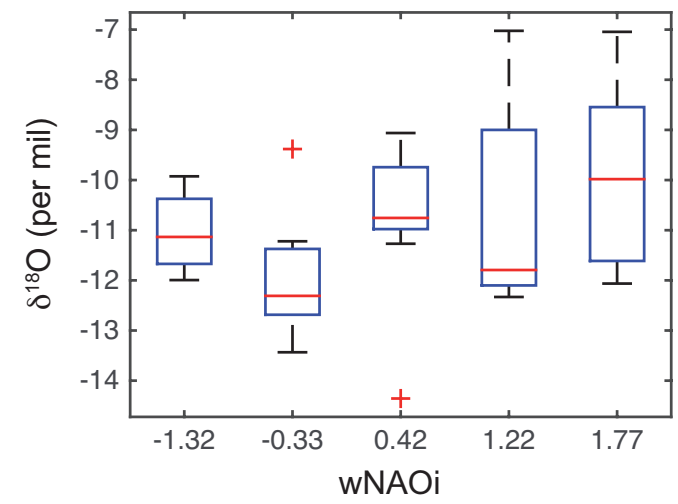

c)

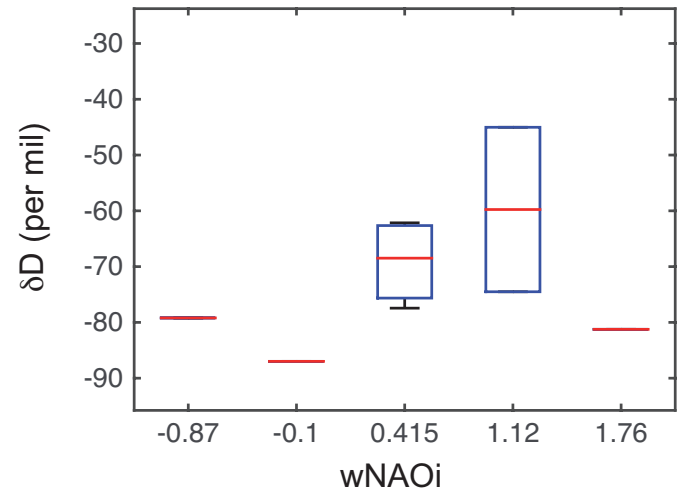

b)

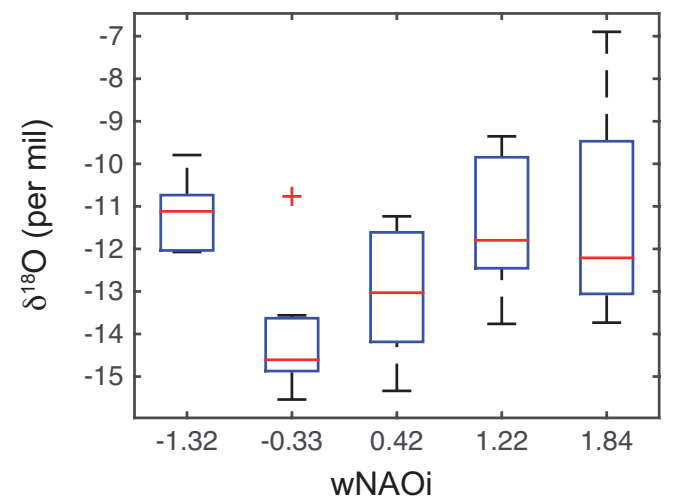

d)

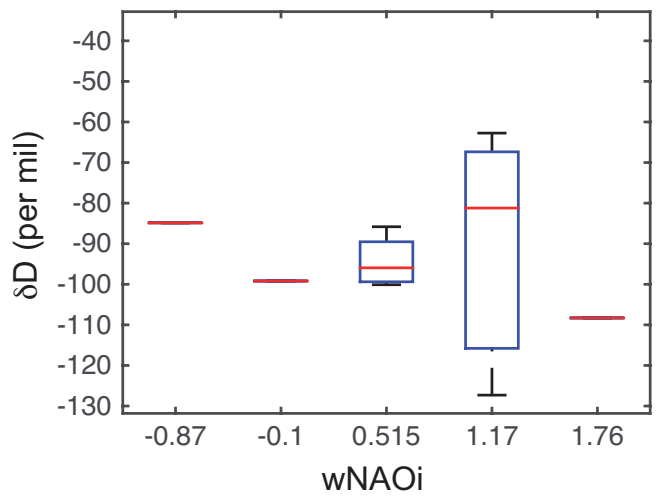

Station 36: Genoa (SESTRI) 
a)

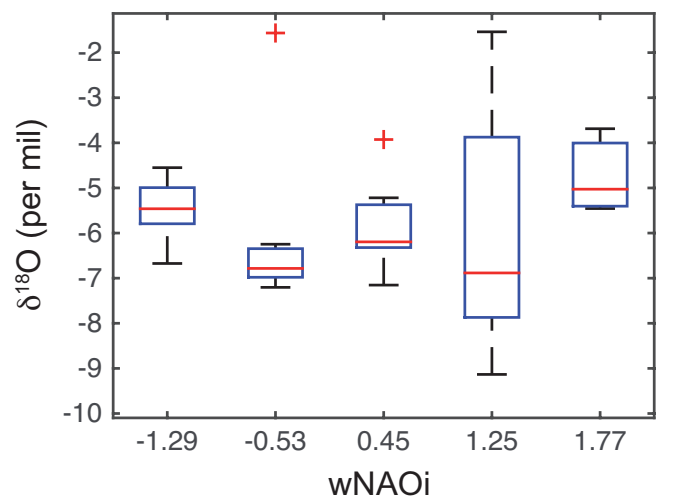

c)

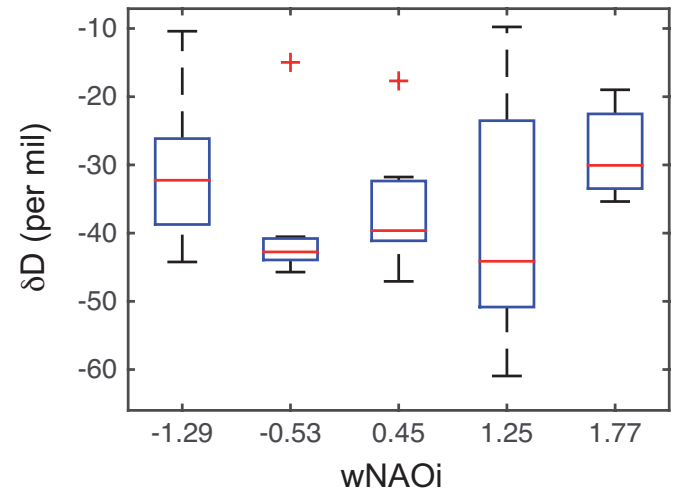

b)

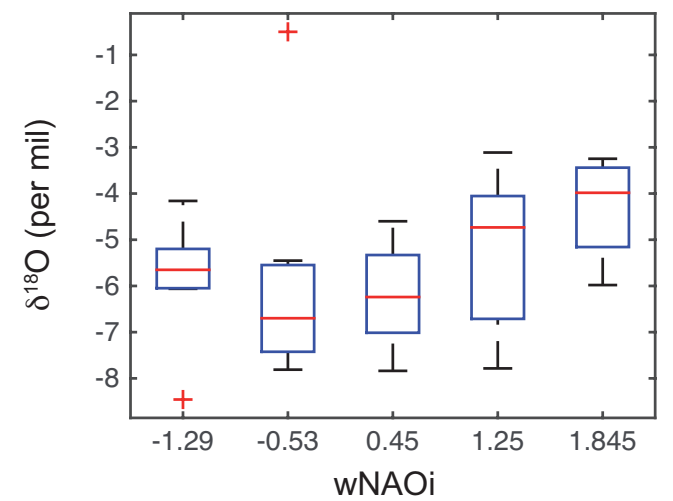

d)

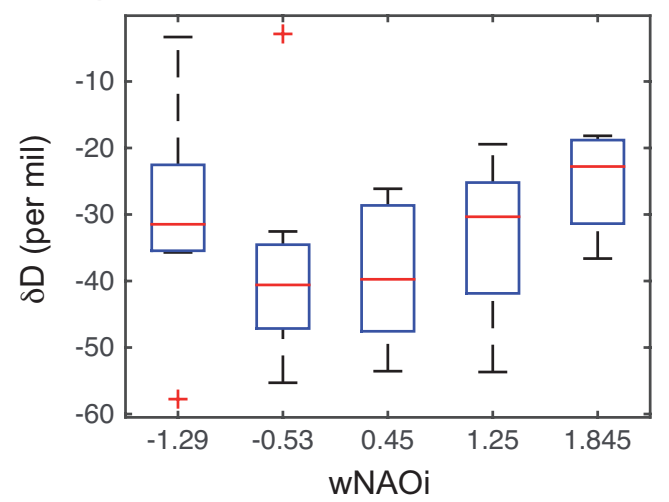

Station 37: Zagreb 
a)

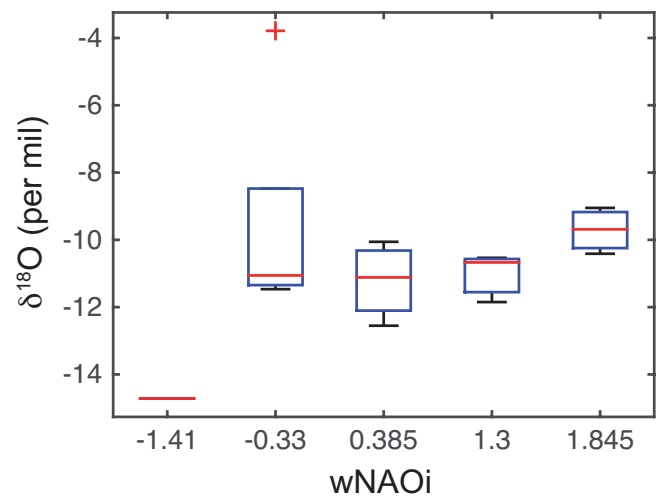

c)

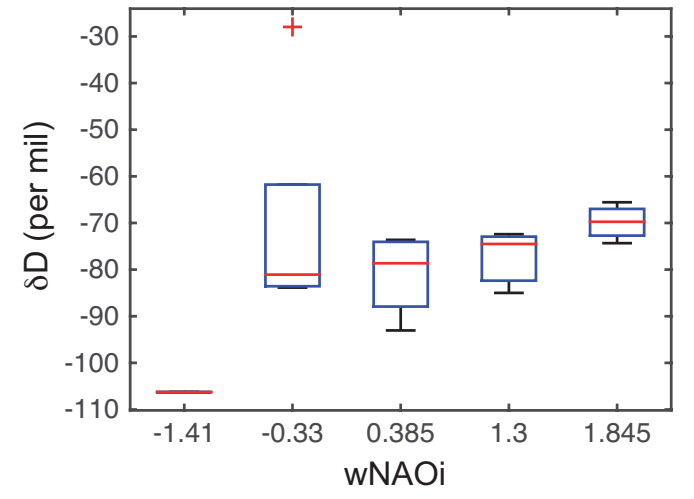

b)

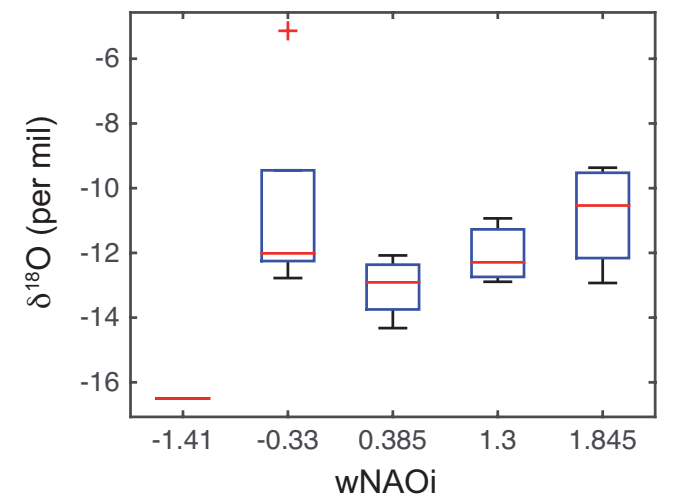

d)

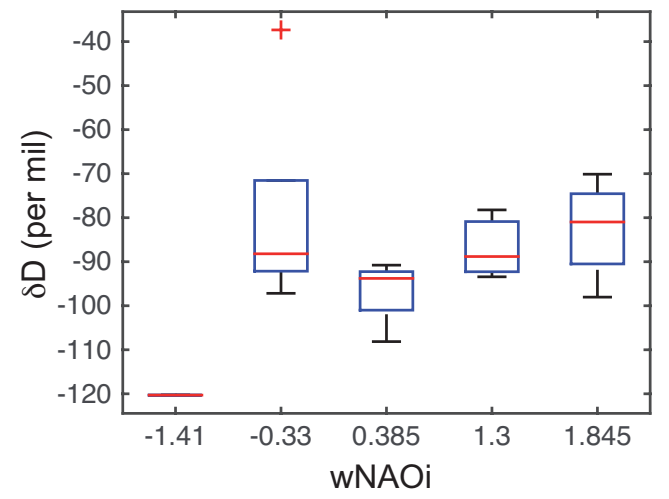

\section{References:}

Majoube, M.: Fractionnement en oxygene-18 et en deuterium entre l'eau et sa vapeur, J. Chim. phys, 68, 1423-1436, 1971.

Mook, W. G.: Introduction to isotope hydrology, Taylor \& Francio, 8, 2006. 